\title{
Hölder Continuity for Sub-Elliptic Systems Under the Sub-Quadratic Controllable Growth in Carnot Groups (*)
}

\author{
JiALin WANG $(* *)(* * *)$ - Dongni Liao $(* * *)-$ Zefeng Yu $(* * *)$
}

ABSTRACT - This paper is devoted to optimal partial regularity of weak solutions to nonlinear sub-elliptic systems for the case $1<m<2$ under the controllable growth condition in Carnot groups. We begin with establishing a Sobolev-Poincaré type inequality for the function $u \in H W^{1, m}\left(\Omega, \mathbb{R}^{N}\right)$ with $m \in(1,2)$, and then partial regularity with optimal local Hölder exponent for horizontal gradients of weak solutions to the systems is established by using $\mathcal{A}$-harmonic approximation technique.

Mathematics Subject Classification (2010). 35H20, 35B65.

KEYwords. Carnot group; Nonlinear sub-elliptic system; Sub-quadratic controllable growth condition; Optimal partial regularity; $\mathcal{A}$-harmonic approximation technique

\section{Introduction and statements of main results}

In this paper, we consider nonlinear sub-elliptic systems of second order in divergence form under the sub-quadratic $(1<m<2)$ controllable growth condition in Carnot groups, and settle optimal partial regularity for horizontal gradients of weak solutions.

More precisely, let $\Omega \subset G$ be a bounded domain in a Carnot group $G$ with general step, and consider the following system

$$
-\sum_{i=1}^{k} X_{i} A_{i}^{\alpha}(\xi, u, X u)=B^{\alpha}(\xi, u, X u), \quad \xi \in \Omega, u \in \mathbb{R}^{N}, X u \in \mathbb{R}^{k N}
$$

(*) Research supported by National Natural Science Foundation of China (No. 11126294 and No. 11201081), supported by Natural Science Foundation of Jiangxi, China (No. 2010GQS0021), and supported by Science and Technology Planning Project of Jiangxi Province, China (No. GJJ13657).

(**) Indirizzo dell'A.: Key Laboratory of Numerical Simulation Technology of Jiangxi Province, Gannan Normal University, Ganzhou, 341000, Jiangxi, P. R. China.

E-mail: jialinwang1025@hotmail.com

(***) Indirizzo degli Autori: School of Mathematics and Computer Science, Gannan Normal University, Ganzhou 341000, Jiangxi, P. R. China. 
with sub-quadratic controllable structure conditions (H1)-(H3) and (C), where

(H1): $A_{i}^{\alpha}(\xi, u, p)$ is differentiable with respect to $p$, with bounded and continuous derivatives, that is, there exists a constant $C$ such that

$$
\left|A_{i, p_{\beta}^{j}}^{\alpha}(\xi, u, p)\right| \leq C\left(1+|p|^{2}\right)^{\frac{m-2}{2}}, \quad(\xi, u, p) \in \Omega \times \mathbb{R}^{N} \times \mathbb{R}^{k N}, 1<m<2,
$$

where we denote by $A_{i, p_{\beta}^{j}}^{\alpha}(\cdot)=\frac{\partial A_{i}^{\alpha}(\cdot)}{\partial p_{\beta}^{j}}$;

(H2): $A_{i}^{\alpha}(\xi, u, p)$ satisfies the following ellipticity condition

$$
A_{i, p_{\beta}^{j}}^{\alpha}(\xi, u, p) \eta_{i}^{\alpha} \eta_{j}^{\beta} \geq \lambda\left(1+|p|^{2}\right)^{\frac{m-2}{2}}|\eta|^{2}, \quad \forall \eta \in \mathbb{R}^{k N},
$$

where $\lambda$ is a positive constant;

(H3): $A_{i}^{\alpha}(\xi, u, p)$ is Hölder continuous with exponent $\gamma \in(0,1)$ in the first and second variables, i.e.,

$$
\left|A_{i}^{\alpha}(\xi, u, p)-A_{i}^{\alpha}(\tilde{\xi}, \tilde{u}, p)\right| \leq K(|u|)\left(d^{m}(\xi, \tilde{\xi})+|u-\tilde{u}|^{m}\right)^{\frac{\gamma}{m}}\left(1+|p|^{2}\right)^{\frac{m-1}{2}},
$$

where $K(\cdot):[0, \infty) \rightarrow[0, \infty)$ is monotonously nondecreasing. Without loss of generality, it is convenient to take $K(\cdot) \geq 1$;

(C) (Controllable growth condition): Denote $r=\frac{m Q}{Q-m}$ and require

$$
\left|B^{\alpha}(\xi, u, p)\right| \leqslant a|p|^{m\left(1-\frac{1}{r}\right)}+b|u|^{r-1}+c,
$$

where $Q \geq 3$ is the homogeneous dimension in Carnot groups (see (2.3) below), and $a, b$ and $c$ are positive constants.

Furthermore, (H1) infers that there exists a continuously nonnegative and bounded function $\omega(s, t):[0, \infty) \times[0, \infty) \rightarrow[0, \infty)$, where $\omega(s, 0)=0$ for all $s$, and $\omega(s, t)$ is monotonously nondecreasing in $s$ for fixed $t ; \omega(s, t)$ is concave and monotonously nondecreasing in $t$ for fixed $s$, such that for all $(\xi, u, p),(\tilde{\xi}, \tilde{u}, \tilde{p}) \in \Omega \times \mathbb{R}^{N} \times \mathbb{R}^{k N}$,

$$
\left|A_{i, p_{\beta}^{j}}^{\alpha}(\xi, u, p)-A_{i, p_{\beta}^{\prime}}^{\alpha}(\xi, u, \tilde{p})\right| \leq C\left(1+|p|^{2}+|\tilde{p}|^{2}\right)^{\frac{m-2}{2}} \omega(|p|,|p-\tilde{p}|) .
$$

As is well known, even under reasonable assumptions on $A_{i}^{\alpha}, B^{\alpha}$ in the systems, people cannot in general expect that weak solutions of nonlinear elliptic systems of equations will be classical (i.e. $C^{2}$-solutions) like elliptic scalar equations. This was first shown by De Giorgi [1]. Then the goal is to establish partial regularity of weak solutions for systems. Such regularity 
means that for any weak solution $u$ of a system, there exists an open subset $\Omega_{0} \subset \Omega$ such that $\Omega \backslash \Omega_{0}$ has a zero Lebesgue measure and $u$ or its gradient $D u$ is locally regular in $\Omega_{0}$. We refer the reader to monographs of Giaquinta [2, 3] and Chen-Wu [4].

There are different methods to prove partial regularity: the direct approach was first carried out by Giaquinta-Modica [5]; Guisti-Miranda [6] was earlier to employ the blow-up method; furthermore, Duzaar and Grotowski in [7] generalized so called $\mathcal{A}$-harmonic approximation technique in [8] and gave the remarkable proof of partial regularity for systems with quadratic growth conditions $(m=2)$. This method has two major advantages: the first is that we only need to establish a Caccioppoli type inequality; this avoids having to prove an inverse Hölder inequality. The other is that one can obtain the optimal Hölder exponent in partial regularity. Then, Duzaar et al. [9] studied partial regularity of almost minimizers of quasi-convex variational integrals with sub-quadratic growth, and we also note that the degenerate $p$-Laplacian version of the method has been obtained in [10], and applied to the partial regularity in [11]. In the paper [9], Duzaar et al. actually provide a partially new proof of the original regularity result of Carozza et al. [12]. Later, Chen and Tan in [13, 14] extended Duzaar and Grotowski's results [7] to more general nonlinear elliptic systems under the super-quadratic growth $(m>2)$ and subquadratic growth $(1<m<2)$, respectively.

Several regularity results were focused on systems constructed by basic vector fields in Carnot groups. Capogna and Garofalo in [15] showed the partial Hölder regularity for quasi-linear sub-elliptic systems under the quadratic structure conditions in Carnot groups of step two. Shores in [16] considered a homogeneous quasi-linear system under the quadratic growth condition on the Carnot group with general step. She first established higher differentiability and smoothness for weak solutions of the system with constant coefficients, and then deduced the partial regularity. Their methods depend mainly on generalization of classical direct method in the Euclidean space. Later, by the method of $\mathcal{A}$-harmonic approximation, Föglein in [17] treated the homogeneous nonlinear system

$$
-\sum_{i=1}^{2 n} X_{i} A_{i}^{\alpha}(\xi, X u)=0, \quad \alpha=1, \cdots, N
$$

on the Heisenberg group under super-quadratic structure conditions. She got partial regularity for the horizontal gradient of weak solutions to the initial system. Then the first author and Niu in [18] considered more gen- 
eral nonlinear sub-elliptic systems (see (1.1)) in Carnot groups under superquadratic growth conditions, and established the optimal partial regularity of the horizontal gradient of weak solutions.

In this paper, we will apply the method of $\mathcal{A}$-harmonic approximation adapted to the setting of Carnot groups to study partial regularity for the system (1.1) under the controllable growth conditions with sub-quadratic case $(1<m<2)$. The key point is to establish a certain excess-decay estimate for the excess function $\Phi$. In the case $m \geq 2$, this function is given by

$$
\Phi\left(\xi_{0}, \rho, p_{0}\right)=\left|B_{\rho}\left(\xi_{0}\right)\right|_{G}^{-1} \int_{B_{\rho}\left(\xi_{0}\right)}\left[\left|X u-p_{0}\right|^{2}+\left|X u-p_{0}\right|^{m}\right] d \xi,
$$

whereas in the case $1<m<2$, one uses

$$
\Phi\left(\xi_{0}, \rho, p_{0}\right)=\left|B_{\rho}\left(\xi_{0}\right)\right|_{G}^{-1} \int_{B_{\rho}\left(\xi_{0}\right)}\left|V(X u)-V\left(p_{0}\right)\right|^{2} d \xi,
$$

where $V(A)=\left(1+|A|^{2}\right)^{\frac{4}{m-2}}$ for $A \in \operatorname{Hom}\left(\mathbb{R}^{k}, \mathbb{R}^{N}\right)$. It is shown that if $\Phi\left(\xi_{0}, \rho, p_{0}\right)$ is small enough on a ball $B_{\rho}\left(\xi_{0}\right) \Subset \Omega$, then for some fixed $\theta \in(0,1)$ one has the excess improvement $\Phi\left(\xi_{0}, \theta \rho, p_{0}\right) \leq C \theta^{2 \gamma} \Phi\left(\xi_{0}, \rho, p_{0}\right)$. Iteration of this result yields the excess-decay estimate which implies the regularity result. Although the underlying philosophy in this paper is encouraged by that in $[14,9]$, some different treatment are necessary. Since basic vector fields (see (2.1) below) of Lie algebras corresponding to the Carnot group are more complicated than gradient vector fields in the Euclidean space, we have to find a different auxiliary function in proving Caccioppoli type inequality. Inspired by [17], we choose horizontal variables to construct such a suitable function (see Remark 1 below); Besides, the non-horizontal derivatives of weak solutions will happen in the Taylor type formula on the Carnot group and cannot been effectively controlled in the present hypotheses. So the method employing Taylor's formula in [14] is not appropriate in our setting. In order to obtain the desired decay estimate, we need establish and use the Sobolev-Poincaré type inequality (3.1) instead.

The main result in this paper is as follows.

THEOREm 1. Assume that coefficients $A_{i}^{\alpha}$ and $B^{\alpha}$ satisfy conditions (H1)-(H3) and (C), and $u \in H W^{1, m}\left(\boldsymbol{\Omega}, \mathbb{R}^{N}\right)$ be a weak solution to the system (1.1) with $\Omega \subset G$, i.e.,

$$
\int_{\Omega} A_{i}^{\alpha}(\xi, u, X u) X_{i} \varphi^{\alpha} d \xi=\int_{\Omega} B^{\alpha}(\xi, u, X u) \varphi^{\alpha} d \xi \quad \forall \varphi \in C_{0}^{\infty}\left(\Omega, \mathbb{R}^{N}\right) .
$$


Then there exists an open subset $\Omega_{0} \subset \Omega$, such that $u \in \Gamma^{1, \gamma}\left(\Omega_{0}, \mathbb{R}^{N}\right)$, with $\gamma$ in (1.4). Furthermore, $\Omega \backslash \Omega_{0}=\Sigma_{1} \cup \Sigma_{2}$ and meas $\left(\Omega \backslash \Omega_{0}\right)=0$, where

$$
\begin{aligned}
& \Sigma_{1}=\left\{\xi_{0} \in \Omega: \lim _{r \rightarrow 0^{+}} \sup \left(\left|(X u)_{\xi_{0}, r}\right|\right)=\infty\right\}, \\
& \Sigma_{2}=\left\{\xi_{0} \in \Omega: \lim _{r \rightarrow 0^{+}} \inf \left|B_{r}\left(\xi_{0}\right)\right|^{-1} \int_{B_{r}\left(\xi_{0}\right)}\left|X u-(X u)_{\xi_{0}, r}\right|^{m} d \xi>0\right\} .
\end{aligned}
$$

To the best of our konwledge, in the Heisenberg group, $\Gamma^{0, \gamma}$ regularity of weak solutions to sub-elliptic $p$-Laplacian equations is valid for $1<p<\infty$ and was proved by several people during the 90 's in [19, 20,21], but when turning to $\Gamma^{1, \gamma}$ regularity, it is worthy of pointing out that the remarkable contribution of Hölder continuity for the gradient of weak solutions to subelliptic $p$-Laplacian equations is due to Capogna [22, 23], Marchi [24, 25], Domokos [26, 27], Manfredi and Mingione et al. [28, 29], but the exponent $p$ should be near 2 , and the limitation $2 \leq p<4$ appears in the most recent of the cited works. Recently, Garofalo in [30] obtained the $\Gamma^{1, \gamma}$ regularity of weak solution which possess some special symmetries for $2 \leq p$. When turning to partial continuity of weak solutions, our result shows that the $\Gamma^{1, \gamma}$ continuity is also valid for exponent $1<p<2$.

The plan of this paper is organized as follows: In Section 2, we introduce two functions and their some useful properties, and collect some basic notions and facts associated to Carnot groups. Since in the subquadratic case we are dealing with functions belonging to the horizontal Sobolev space $H W^{1, m}$ with $1<m<2$, in the proof of the main results, a Sobolev-Poincaré type inequality (see (3.1) below) will be used. So we will prove this inequality and a prior estimate for constant systems in Section 3. In Section 4, we show a Caccioppoli type inequality for weak solutions to the system (1.8). Section 5 is devoted to the proof of Theorem 1, and the process of the proof is split into four steps. An estimate on weak solutions is established in the first step. Second step begins with proving three "small conditions" (see (5.13)-(5.15) below), and then obtain the excess-improvement for the function $\Phi$ in (1.7). Third step is to show that we can iterate the statement in the second step. A decay estimate

$$
\Phi\left(\xi_{0}, \rho, p_{0}\right) \leq C \rho^{2 \gamma}
$$

is obtained in the last step, where $\gamma$ is the same as (1.4). Then we can infer the conclusion of Theorem 1 employing Lemma 2 and Lemma 3 below. 


\section{Preliminaries}

A Carnot group $G$ of step $r$ is a simply connected, nilpotent Lie group whose Lie algebra $\widetilde{g}$ admits a stratification, i.e., $\widetilde{g}=\bigoplus_{j=1}^{r} V^{j}$ such that $\left[V^{1}, V^{j}\right]=V^{j+1}, j=1, \cdots, r-1$ and $\left[V^{1}, V^{r}\right]=\{0\}$. Let $X_{i}^{l}$ denote a leftinvariant basis vector field of $V^{l}$ with $1 \leq l \leq r$ and $1 \leq i \leq m_{l}$, where $m_{l}$ is the dimension of $V^{l}$. For the sake of simplicity, we let $X_{i}=X_{i}^{1}, k=m_{1}$, and denote by $X=\left(X_{1}, \cdots, X_{k}\right)$ the sub-elliptic gradient. We will say that $X_{i}(i=1, \cdots, k)$ are the horizontal vector fields with the form

$$
X_{i}=\partial_{i}+\sum_{j=i+1}^{n} a_{i j}(\xi) \partial_{j}, \quad X_{i}(0)=\partial_{i},
$$

where $a_{i j}(\xi)$ is a polynomial. For a vector valued function $u=\left(u^{1}, \cdots, u^{N}\right): G \mapsto \mathbb{R}^{N}$, we let $X_{i} u^{\alpha}(i=1, \cdots, k, \alpha=1, \cdots, N)$ be a horizontal direction derivative, and say that $X u$ is the horizontal Jacobian with entries $X_{i} u^{\alpha}$.

Denoting

$$
\xi=\left(\xi^{1}, \xi^{2}, \cdots, \xi^{r}\right)=\left(x_{1}^{1}, x_{2}^{1}, \cdots, x_{m_{1}}^{1} ; x_{1}^{2}, \cdots, x_{m_{2}}^{2} ; \cdots ; x_{1}^{r}, \cdots, x_{m_{l}}^{r}\right) \in G,
$$

the distance from origin defined by

$$
d(\xi)=\left[\sum_{l=1}^{r}\left(\sum_{i=1}^{m_{l}}\left|x_{i}^{l}\right|^{2}\right)^{\frac{r !}{l}}\right]^{\frac{1}{2 ! !}} .
$$

For any $\xi, \eta \in G$, we set $d(\xi, \eta)=d\left(\eta^{-1} \circ \xi\right)$, where $\eta^{-1}=-\eta=$ $\left(-\eta^{1}, \cdots,-\eta^{r}\right)$ is the reverse of $\eta$, and $\circ$ is the multiplication rule in $G$ defined by $\xi \circ \widetilde{\xi}=\xi+\widetilde{\xi}+P(\xi, \widetilde{\xi}), \quad \xi, \widetilde{\xi} \in G$, where $P: G \times G \mapsto G$ has polynomial components.

Following [31], we introduce the gauge ball and sphere $B_{r}(\xi)=$ $\{\eta \in G \mid d(\xi, \eta)<r\}, \partial B_{r}(\xi)=\{\eta \in G \mid d(\xi, \eta)=r\}$ in $G$, respectively. In what follows, we denote by $\omega_{G}=|B(0,1)|_{G}$ the volume of unit ball. Then $|B(\xi, r)|_{G}=\omega_{G} r^{Q}$, where

$$
Q=\sum_{l=1}^{r} l m_{l}
$$

is the homogeneous dimension of $G$. 
DeFinition 1. Let $1 \leq m<\infty$, and $\Omega \subset G$ be an open set. If $u \in L^{m}(\Omega)$ satisfies

$$
\|u\|_{H W^{1, m}(\Omega)} \equiv\|u\|_{L^{m}(\Omega)}+\sum_{i=1}^{k}\left\|X_{i} u\right\|_{L^{m}(\Omega)}<\infty
$$

we say that $u$ belongs to the horizontal Sobolev space. The space $H W_{0}^{1, m}(\Omega)$ is the completion of $C_{0}^{\infty}(\Omega)$ under the norm (2.4).

Definition 2. Let $\Omega \subset G, \gamma \in(0,1)$, and denote

$$
\Gamma^{1, \gamma}(\Omega)=\left\{u \in L^{\infty}(\Omega) \mid X_{i} u \in \Gamma^{\gamma}(\Omega), i=1, \cdots, k\right\},
$$

where $\Gamma^{\gamma}(\Omega)=\left\{\left.u \in L^{\infty}(\Omega)|| u\right|_{\gamma}=\sup _{\xi, \tilde{\xi} \in \Omega, \xi \neq \tilde{\xi}} \frac{|u(\xi)-u(\tilde{\xi})|}{d(\xi, \tilde{\xi})^{\gamma}}<\infty\right\}$. We say that $\Gamma^{1, \gamma}(\Omega)$ is a Folland-Stein space with the norm

$$
\|u\|_{\Gamma^{1, \gamma}(\Omega)}=\|u\|_{L^{\infty}(\Omega)}+\|X u\|_{L^{\infty}(\Omega)}+|u|_{\gamma}+|X u|_{\gamma} .
$$

Definition 3. Let $\Omega \subset G, 1 \leq m<\infty$ and $\mu \geq 0$. We say that $u \in L^{m}(\Omega)$ belongs to the Campanato space $\mathcal{L}^{m, \mu}(\Omega)$ if

$$
\|u\|_{\mathcal{L}^{m, \mu}(\Omega)}=\|u\|_{L^{m}(\Omega)}+\left(\sup _{\xi \in \Omega, \rho<\operatorname{diam} \Omega} \rho^{-\mu} \int_{B_{\rho}(\xi) \cap \Omega}\left|u(\zeta)-u_{\xi, \rho}\right|^{m} d \zeta\right)^{\frac{1}{m}}<\infty .
$$

Capogna in [23] proved a Campanato type lemma in Carnot groups.

Lemma 2. Let $\xi \in \Omega \subset G, m \geq 1, r>0$, and $Q<\mu \leq Q+m$, then

$$
\mathcal{L}^{m, \mu}\left(B_{\rho}(\xi)\right) \subset \Gamma^{\gamma}\left(B_{\rho}(\xi)\right), \gamma=\frac{\mu-Q}{m} .
$$

Throughout the paper, we shall use the functions $V=V_{m}$, $W=W_{m}: \mathbb{R}^{n} \rightarrow \mathbb{R}^{n}$ defined by

$$
V(\varsigma)=\varsigma /\left(1+|\varsigma|^{2}\right)^{\frac{2-m}{4}}, \quad W(\varsigma)=\varsigma /\left(1+|\varsigma|^{2-m}\right)^{\frac{1}{2}}
$$

for each $\varsigma \in \mathbb{R}^{n}$ and $m>1$. By the elementary inequality $\|x\|_{\frac{2}{2-m}} \leq\|x\|_{1} \leq$ $2^{\frac{m}{2}}\|x\|_{\frac{2}{2-m}}$ applied to the vector $x=\left(1,|\varsigma|^{2-m}\right) \in \mathbb{R}^{2}$ we conclude that

$$
\left(1+|\varsigma|^{2}\right)^{\frac{2-m}{2}} \leq 1+|\varsigma|^{2-m} \leq 2^{\frac{m}{2}}\left(1+|\varsigma|^{2}\right)^{\frac{2-m}{2}},
$$


which immediately yields

$$
|W(\varsigma)| \leq|V(\varsigma)| \leq 2^{\frac{m}{4}}|W(\varsigma)| .
$$

The purpose of introducing $W$ is the fact that in contrast to $|V|^{\frac{2}{m}}$, the function $|W|^{\frac{2}{m}}$ is convex (see [9]).

The following lemma includes some useful properties of the function $V$, which can be found in Lemma 2.1 of [12].

Lemma 3. Let $m \in(1,2)$ and $V, W: \mathbb{R}^{n} \rightarrow \mathbb{R}^{n}$ be the functions defined in (2.8). Then there holds for any $\varsigma_{1}, \varsigma_{2} \in \mathbb{R}^{n}$ and $t>0$ :
(1). $\frac{1}{\sqrt{2}} \min \left(\left|\varsigma_{1}\right|,\left|\varsigma_{1}\right|^{\frac{m}{2}}\right) \leq\left|V\left(\varsigma_{1}\right)\right| \leq \min \left(\left|\varsigma_{1}\right|,\left|\varsigma_{1}\right|^{\frac{m}{2}}\right)$;
(2). $\left|V\left(t_{\varsigma_{1}}\right)\right| \leq \max \left(t, t^{\frac{m}{2}}\right)\left|V\left(\varsigma_{1}\right)\right|$;
(3). $\left|V\left(\varsigma_{1}+\varsigma_{2}\right)\right| \leq C(m)\left(\left|V\left(\varsigma_{1}\right)\right|+\left|V\left(\varsigma_{2}\right)\right|\right)$;
(4). $\frac{m}{2}\left|\varsigma_{1}-\varsigma_{2}\right| \leq\left|V\left(\varsigma_{1}\right)-V\left(\varsigma_{2}\right)\right| /\left(1+\left|\varsigma_{1}\right|^{2}+\left|\varsigma_{2}\right|^{2}\right)^{\frac{m-2}{4}} \leq C(m, n)\left|\varsigma_{1}-\varsigma_{2}\right|$;
(5). $\left|V\left(\varsigma_{1}\right)-V\left(\varsigma_{2}\right)\right| \leq C(m, n)\left|V\left(\varsigma_{1}-\varsigma_{2}\right)\right|$;
(6). $\left|V\left(\varsigma_{1}-\varsigma_{2}\right)\right| \leq C(m, M)\left|V\left(\varsigma_{1}\right)-V\left(\varsigma_{2}\right)\right|$ for all $\varsigma_{1}$ with $\left|\varsigma_{2}\right| \leq M$.

The inequalities (1)-(3) also hold if $V$ is replaced by $W$.

For later purpose we state the following two simple estimates which can be easily deduced from Lemma 3 (1) and (6). Let $\varsigma_{1}, \varsigma_{2} \in \mathbb{R}^{n}$ with $\left|\varsigma_{2}\right| \leq M$ it follows that

$$
\left|\varsigma_{1}-\varsigma_{2}\right|^{2} \leq C(m, M)\left|V\left(\varsigma_{1}\right)-V\left(\varsigma_{2}\right)\right|^{2},
$$

for $\left|\varsigma_{1}-\varsigma_{2}\right| \leq 1$, while for $\left|\varsigma_{1}-\varsigma_{2}\right|>1$

$$
\left|\varsigma_{1}-\varsigma_{2}\right|^{m} \leq C(m, M)\left|V\left(\varsigma_{1}\right)-V\left(\varsigma_{2}\right)\right|^{2} .
$$

The next lemma due to [9] is a more general version of the Lemma 2.7 in [12].

Lemma 4. Let $0 \leq v<1, a, b \geq 0, v \in L^{p}\left(B_{\rho}\left(\xi_{0}\right)\right)$ and $g$ be a non-negative bounded function satisfying

$$
g(t) \leq v g(s)+a \int_{B_{\rho}\left(\xi_{0}\right)}\left|V\left(\frac{v}{s-t}\right)\right|^{2} d \xi+b,
$$


for all $\frac{\rho}{2} \leq t<s \leq \rho$. Then there exists a constant $C=C(v)$ such that

$$
g\left(\frac{\rho}{2}\right) \leq C(v)\left(a \int_{B_{\rho}\left(\xi_{0}\right)}\left|V\left(\frac{v}{\rho}\right)\right|^{2} d \xi+b\right) .
$$

We conclude this section with an algebraic fact from [32]

Lemma 5. For every $t \in\left(-\frac{1}{2}, 0\right)$ and $\mu \geq 0$, it holds

$$
1 \leq \frac{\int_{0}^{1}\left(\mu^{2}+|p+s(\tilde{p}-p)|^{2}\right)^{t} d s}{\left(\mu^{2}+|p|^{2}+|\tilde{p}|^{2}\right)^{t}} \leq \frac{8}{2 t+1}
$$

for any $p, \tilde{p} \in R^{k N}$, not both zero if $\mu=0$.

\section{Sobolev-Poincaré type inequality and a prior estimate in the sub- quadratic case}

LEMma 6 (Sobolev-Poincaré type inequality). Let $m \in(1,2)$ and $u \in H W^{1, m}\left(B_{\rho}\left(\xi_{0}\right), \mathbb{R}^{N}\right)$ with $B_{\rho}\left(\xi_{0}\right) \subset \Omega$, then

$$
\begin{aligned}
\left(\left|B_{\rho}\left(\xi_{0}\right)\right|_{G}^{-1} \int_{B_{\rho}\left(\xi_{0}\right)}\left|W\left(\frac{u-u_{\xi_{0}, \rho}}{\rho}\right)\right|^{\frac{2 Q}{Q-m}} d \xi\right)^{\frac{Q-m}{2 Q}} \\
\leq C_{P}\left(\left|B_{\rho}\left(\xi_{0}\right)\right|_{G}^{-1} \int_{B_{\rho}\left(\xi_{0}\right)}|W(X u)|^{2} d \xi\right)^{\frac{1}{2}}
\end{aligned}
$$

Furthermore, the analogous inequality is valid with $W$ replaced by $V$ defined in (2.8), and in particular, the inequality also holds if we substitute 2 for $\frac{2 Q}{Q-m}$.

Proof. Similarly to the proof of Theorem 2 in [9]. Following [33, Lemma 3.2], there exist $c>1$ and $C>1$ such that for $\xi \in B\left(\xi_{0}, \rho\right)$

$$
\left|u(\xi)-u_{\xi_{0}, \rho}\right| \leq C \int_{B_{c \rho}\left(\xi_{0}\right)} \frac{|X u(\eta)|}{d(\xi, \eta)^{Q-1}} d \eta
$$


Since $W^{2 / m}(t)$ is non-decreasing and convex, we apply $W^{2 / m}(t)$ to both sides of the last inequality and have by Jensen's inequality and recalling $W(0)=0$ :

$$
W^{2 / m}\left(\left|\frac{u(\xi)-u_{\xi_{0}, \rho}}{\rho}\right|\right) \leq \frac{C}{\rho} \int_{G} \mathrm{~d}^{1-Q}(\xi, \eta) \widetilde{W}^{2 / m}(|X u(\eta)|) d \eta,
$$

where the number $Q$ is the homogeneous dimension in $G$, and

$$
\widetilde{W}(|X u(\eta)|)= \begin{cases}0, & \eta \notin B_{c \rho}\left(\xi_{0}\right), \\ W(|X u(\eta)|), & \eta \in B_{c \rho}\left(\xi_{0}\right) .\end{cases}
$$

Since $W(|X u(\eta)|) \in L^{m}\left(B_{\rho}\left(\xi_{0}\right)\right)$ by Lemma 3(1), it implies $\widetilde{W}(|X u(\eta)|) \in$ $L^{m}(G)$. Then Theorem 2.7 in [19] applied with $\widetilde{W}^{2 / m}(|X u|), \alpha=1$ yields

$$
\begin{aligned}
& {\left[\int_{B_{\rho}\left(\xi_{0}\right)}\left|W\left(\frac{u(\xi)-u_{\xi_{0}, \rho}}{\rho}\right)\right|^{\frac{2 Q}{Q-m}} d \xi\right]^{\frac{Q-m}{m Q}} } \\
= & {\left[\int_{B_{\rho}\left(\xi_{0}\right)} W^{2 / m}\left(\frac{\left|u(\xi)-u_{\xi_{0}, \rho}\right|}{\rho}\right)^{\frac{m Q}{Q-m}} d \xi\right]^{\frac{Q-m}{m Q}} } \\
\leq & \frac{C}{\rho}\left[\int_{G}\left(\int_{G} d^{1-Q}(\xi, \eta) \widetilde{W}^{2 / m}(|X u(\eta)|) d \eta\right)^{\frac{m Q}{Q-m}} d \xi\right]^{\frac{Q-m}{m Q}} \\
\leq & \frac{C}{\rho}\left[\int_{G} \widetilde{W}^{2 / m}(|X u(\xi)|)^{m} d \xi\right]^{\frac{1}{m}} \\
= & \frac{C}{\rho}\left[\int_{B_{\rho}\left(\xi_{0}\right)} W^{2}(|X u(\xi)|) d \xi\right]^{\frac{1}{m}},
\end{aligned}
$$

or

$$
\left[\int_{B_{\rho}\left(\xi_{0}\right)}\left|W\left(\frac{u(\xi)-u_{\xi_{0}, \rho}}{2 \rho}\right)\right|^{\frac{2 Q}{Q-m}} d \xi\right]^{\frac{Q-m}{2 Q}} \leq C \rho^{-\frac{m}{2}}\left[\int_{B_{\rho}\left(\xi_{0}\right)} W^{2}(|X u(\xi)|) d \xi\right]^{\frac{1}{2}}
$$

Note that $W(\varsigma / 2) \geq W(\varsigma) / 2$, we conclude the result (3.1) from (3.4). 
Lemma 7. Let $u \in H W^{1,1}\left(\Omega, \mathbb{R}^{N}\right)$ be a weak solution of

$$
\int_{\Omega} A_{i j}^{\alpha \beta} X_{j} u^{\beta} X_{i} \phi^{\alpha} d \xi=0
$$

for any $\phi \in C_{0}^{1}\left(\Omega, \mathbb{R}^{N}\right)$, where $A_{i j}^{\alpha \beta}$ is a constant matrix satisfying the strong Legendre-Hadamard condition:

$$
A_{i j}^{\alpha \beta} \eta^{\alpha} \eta^{\beta} \mu_{i} \mu_{j}>c|\eta|^{2}|\mu|^{2}, \quad \eta \in \mathbb{R}^{N}, \mu \in \mathbb{R}^{k} .
$$

Then $u$ is smooth and there exists $C_{0} \geq 1$ such that for any $B_{\rho}\left(\xi_{0}\right) \subset \Omega$

$$
\sup _{B_{\rho / 2}\left(\xi_{0}\right)}\left(\left|u-u_{\xi_{0}, \rho}\right|^{2}+\rho^{2}|X u|^{2}+\rho^{4}\left|X^{2} u\right|^{2}\right) \leq C_{0}\left|B_{\rho}\left(\xi_{0}\right)\right|_{G}^{-1} \rho^{2} \int_{B_{\rho}\left(\xi_{0}\right)}|X u|^{2} d \xi .
$$

PRoof. Shores [16] has shown that a weak solution $v \in H W^{1,2}\left(\Omega, \mathbb{R}^{N}\right)$ of (3.5) belongs to $C^{\infty}$ in the subset $\Omega_{0} \subset \Omega$, and Wang and Niu [18] have established the following estimate:

$$
\sup _{B_{\rho / 2}\left(\xi_{0}\right)}\left(\left|v-v_{\xi_{0}, \rho}\right|^{2}+\rho^{2}|X v|^{2}+\rho^{4}\left|X^{2} v\right|^{2}\right) \leq C_{0}\left|B_{\rho}\left(\xi_{0}\right)\right|_{G}^{-1} \rho^{2} \int_{B_{\rho}\left(\xi_{0}\right)}|X v|^{2} d \xi .
$$

Then we can argue as in the proof of Proposition 2.10 in [12] to obtain the conclusion.

\section{Caccioppoli type inequality}

Denote by Bil $\left(\mathbb{R}^{k N}\right)$ the collection of bi-linear forms defined in $\mathbb{R}^{k N}$, and suppose $\mathcal{A} \in \operatorname{Bil}\left(\mathbb{R}^{k N}\right)$. We say that a function $h \in H W^{1, m}\left(\Omega, \mathbb{R}^{N}\right)$ is $\mathcal{A}$-harmonic, if $h$ satisfies

$$
\int_{\Omega} \mathcal{A}(X h, X \varphi) d \xi=0, \quad \forall \varphi \in C_{0}^{1}\left(\Omega, \mathbb{R}^{N}\right) .
$$

Similarly to [9], one can establish the following $\mathcal{A}$-harmonic approximation lemma for the case $1<m<2$ in Carnot groups.

Lemma 8. Let $\lambda$ and $L$ be fixed positive numbers, $1<m<2$, and $k, N \in \mathbb{N}$ with $k \geq 2$. If for any given $\varepsilon>0$, there exists $\delta=$ $\delta(k, N, \lambda, L, \varepsilon) \in(0,1]$ with the following properties: 
(I) for any $\mathcal{A} \in \operatorname{Bil}\left(\mathbb{R}^{k N}\right)$ satisfying

$$
\mathcal{A}(v, v) \geq \lambda|v|^{2} \text { and } \mathcal{A}(v, \bar{v}) \leq L|v||\bar{v}|, \quad v, \bar{v} \in \mathbb{R}^{k N},
$$

(II) for any $g \in H W^{1, m}\left(B_{\rho}\left(\xi_{0}\right), \mathbb{R}^{N}\right)$ satisfying

$$
\left|B_{\rho}\left(\xi_{0}\right)\right|_{G}^{-1} \int_{B_{\rho}\left(\xi_{0}\right)}|W(X g)|^{2} d \xi \leq \Upsilon^{2} \leq 1 \text { and }
$$

$$
\left.|| B_{\rho}\left(\xi_{0}\right)\right|_{G} ^{-1} \int_{B_{\rho}\left(\xi_{0}\right)} \mathcal{A}(X g, X \varphi) d \xi\left|\leq \Upsilon \delta \sup _{B_{\rho}\left(\xi_{0}\right)}\right| X \varphi \mid, \quad \forall \varphi \in C_{0}^{1}\left(B_{\rho}\left(\xi_{0}\right),\left\{\mathbb{R}^{N}\right)\right.
$$

then there exists an $\mathcal{A}$-harmonic function $h$

$$
h \in H=\left\{h \in H W^{1, m}\left(B_{\rho}\left(\xi_{0}\right),\left.\left\{\mathbb{R}^{N}\right)|| B_{\rho}\left(\xi_{0}\right)\right|_{G} ^{-1} \int_{B_{\rho}\left(\xi_{0}\right)}|W(X h)|^{2} d \xi \leq 1\right\}\right.
$$

such that

$$
\left|B_{\rho}\left(\xi_{0}\right)\right|_{G}^{-1} \int_{B_{\rho}\left(\xi_{0}\right)}\left|W\left(\frac{g-\Upsilon h}{\rho}\right)\right|^{2} d \xi \leq \Upsilon^{2} \varepsilon
$$

To establish the main result, our first aim is to establish a suitable Caccioppoli type inequality.

LEMMA 9 (Caccioppoli Type Inequality). Let $u \in H W^{1, m}\left(\Omega,\left\{\mathbb{R}^{N}\right)\right.$ be a weak solution to the system (1.1) under the conditions (H1)-(H3) and (C). Then for every $\xi_{0}=\left(\xi_{0}^{1}, \cdots, \xi_{0}^{r}\right) \in \Omega, u_{0} \in \mathbb{R}^{N}, p_{0} \in\left\{\mathbb{R}^{k N}\right.$ and any $\rho$ satisfying $0<\rho<\min \left\{1, \operatorname{dist}\left(\xi_{0}, \partial \Omega\right)\right\}$, it holds

$$
\begin{aligned}
& \left|B_{\rho / 2}\left(\xi_{0}\right)\right|_{G}^{-1} \int_{B_{\rho / 2}\left(\xi_{0}\right)}\left|V\left(X u-p_{0}\right)\right|^{2} d \xi \\
& \leq C_{c}\left\{\left|B_{\rho}\left(\xi_{0}\right)\right|_{G}^{-1} \int_{B_{\rho}\left(\xi_{0}\right)}\left|V\left(\frac{u-u_{0}-p_{0}\left(\xi^{1}-\xi_{0}^{1}\right)}{\rho}\right)\right|^{2} d \xi\right.
\end{aligned}
$$

$\left.+\left[K(\cdot)\left(1+\left|p_{0}\right|^{m / 2}\right)\right]^{\sigma} \rho^{2 \gamma}\left|+B_{\rho}\left(\xi_{0}\right)\right|_{G}^{-1}\left[\int_{B_{\rho}\left(\xi_{0}\right)}\left(|X u|^{m}+|u|^{r}+1\right) d \xi\right]^{\frac{m}{m-1}\left(1-\frac{1}{r}\right)}\right\}$ 
where we denote $K(\cdot)=K\left(\left|u_{0}\right|+\left|p_{0}\right|\right), \xi^{1}=\left(\xi_{1}^{1}, \xi_{2}^{1}, \cdots, \xi_{k}^{1}\right)$ is the horizontal component of $\xi=\left(\xi^{1}, \cdots, \xi^{r}\right) \in G, \sigma=\max \{2 m /(m-2 \gamma), m /(m-1-\gamma)\}>2$ and the constant $C_{c}=C_{c}(a, b, c, n, N, m, L, \lambda, M)$.

Proof. Let $B_{\rho}\left(\xi_{0}\right) \subset \subset \Omega, \rho / 2 \leq t<s \leq \rho$, and a standard cut off function $\eta \in C_{0}^{\infty}\left(B_{s}\left(\xi_{0}\right)\right)$ satisfy $0 \leq \eta \leq 1,|\nabla \eta|<\frac{C}{s-t}$ and $\eta \equiv 1$ on $B_{t}\left(\xi_{0}\right)$. Inspired by the way of [17], we let $v=u(\xi)-u_{0}-p_{0}\left(\xi^{1}-\xi_{0}^{1}\right)$, and then define two functions

$$
\varphi=\eta v, \quad \phi=(1-\eta) v
$$

one has

$$
X \varphi+X \phi=X u-p_{0}
$$

and

$$
|X \varphi|^{m},|X \phi|^{m} \leq C(m)\left(|X v|^{m}+\left|\frac{v}{s-t}\right|^{m}\right) .
$$

Using hypothesis (H2), from Lemma 5, and as the elementary inequality

$$
1+|a|^{2}+|b-a|^{2} \leq 3\left(1+|a|^{2}+|b|^{2}\right),
$$

we have

$$
\begin{aligned}
& \int_{B_{s}\left(\xi_{0}\right)}\left[A_{i}^{\alpha}\left(\xi, u, p_{0}+X \varphi\right)-A_{i}^{\alpha}\left(\xi, u, p_{0}\right)\right] X_{i} \varphi^{\alpha} d \xi \\
= & \int_{B_{s}\left(\xi_{0}\right)}\left[\int_{0}^{1} \frac{d A_{i}^{\alpha}\left(\xi, u, p_{0}+\theta X \varphi\right)}{d \theta} d \theta\right] X_{i} \varphi^{\alpha} d \xi \\
= & \int_{B_{s}\left(\xi_{0}\right)} \int_{0}^{1} \frac{\partial A_{i}^{\alpha}\left(\xi, u, p_{0}+\theta X \varphi\right)}{\partial p_{j}^{\beta}} d \theta X_{j} \varphi^{\beta} X_{i} \varphi^{\alpha} d \xi \\
\geq & \lambda \int_{B_{s}\left(\xi_{0}\right)} \int_{0}^{1}\left[1+\left|p_{0}+\theta\left(\left(X \varphi+p_{0}\right)-p_{0}\right)\right|^{2}\right]^{(m-2) / 2} d \theta|X \varphi|^{2} d \xi \\
\geq & \int_{B_{s}\left(\xi_{0}\right)} \int_{\left(1+\left|p_{0}\right|^{2}+\left|X \varphi-p_{0}\right|^{2}\right)^{(m-2) / 2}|X \varphi|^{2} d \xi} 3^{(m-2) / 2} \int_{B_{s}\left(\xi_{0}\right)}\left(1+\left|p_{0}\right|^{2}+|X \varphi|^{2}\right)^{(m-2) / 2}|X \varphi|^{2} d \xi .
\end{aligned}
$$


From (4.11), it follows that

$$
\begin{aligned}
& 3^{(m-2) / 2} \lambda \int_{B_{s}\left(\xi_{0}\right)}\left(1+\left|p_{0}\right|^{2}+|X \varphi|^{2}\right)^{(m-2) / 2}|X \varphi|^{2} d \xi \\
& \leq \int_{B_{s}\left(\xi_{0}\right)}\left[A_{i}^{\alpha}\left(\xi, u, p_{0}+X \varphi\right)-A_{i}^{\alpha}\left(\xi, u, p_{0}\right)\right] X_{i} \varphi^{\alpha} d \xi \\
& =\int_{B_{s}\left(\xi_{0}\right)} A_{i}^{\alpha}(\xi, u, X u) X_{i} \varphi^{\alpha} d \xi-\int_{B_{s}\left(\xi_{0}\right)} A_{i}^{\alpha}\left(\xi, u, p_{0}\right) X_{i} \varphi^{\alpha} d \xi \\
& +\int_{B_{s}\left(\xi_{0}\right)}\left[A_{i}^{\alpha}\left(\xi, u, p_{0}+X \varphi\right)-A_{i}^{\alpha}(\xi, u, X u)\right] X_{i} \varphi^{\alpha} d \xi \\
& \text { (4.12) } \leq-\int_{B_{s}\left(\xi_{0}\right)}\left[A_{i}^{\alpha}\left(\xi, u, p_{0}\right)-A_{i}^{\alpha}\left(\xi, u_{0}+p_{0}\left(\xi^{1}-\xi_{0}^{1}\right), p_{0}\right)\right] X_{i} \varphi^{\alpha} d \xi \\
& -\int_{B_{s}\left(\xi_{0}\right)}\left[A_{i}^{\alpha}\left(\xi, u_{0}+p_{0}\left(\xi^{1}-\xi_{0}^{1}\right), p_{0}\right)-A_{i}^{\alpha}\left(\xi_{0}, u_{0}, p_{0}\right)\right] X_{i} \varphi^{\alpha} d \xi \\
& -\int_{B_{s}\left(\xi_{0}\right)} \int_{0}^{1} \frac{\partial A_{i}^{\alpha}\left(\xi, u, X u-\theta\left(X u-X \varphi-p_{0}\right)\right)}{\partial p_{j}^{\beta}} d \theta X_{j} \phi^{\beta} X_{i} \varphi^{\alpha} d \xi \\
& +\int_{B_{s}\left(\xi_{0}\right)} B_{i}(\xi, u, X u) \varphi^{\alpha} d \xi \\
& :=I+I I+I I I+I V \text {, }
\end{aligned}
$$

where we have used (4.8), (1.8) and the fact that

$$
\int_{B_{R}\left(\xi_{0}\right)} A_{i}^{\alpha}\left(\xi_{0}, u_{0}, p_{0}\right) X \varphi^{\alpha} d \xi=0
$$

Noting that $\varphi=v$ on $B_{t}\left(\xi_{0}\right)$, and (4.10), the left-hand side in (4.12) can be estimated by

$$
\begin{aligned}
& 3^{(m-2) / 2} \lambda \int_{B_{t}\left(\xi_{0}\right)}\left(1+\left|p_{0}\right|^{2}+|X \varphi|^{2}\right)^{(m-2) / 2}|X \varphi|^{2} d \xi \\
= & 3^{(m-2) / 2} \lambda \int_{B_{t}\left(\xi_{0}\right)}\left(1+\left|p_{0}\right|^{2}+|X v|^{2}\right)^{(m-2) / 2}|X v|^{2} d \xi
\end{aligned}
$$




$$
\begin{aligned}
& \geq C(m) \lambda \int_{B_{t}\left(\xi_{0}\right)}\left[\left(1+\left|p_{0}\right|^{2}+|X u|^{2}\right)^{(m-2) / 4}\left|X u-p_{0}\right|\right]^{2} d \xi \\
& \geq C(k, N, m, \lambda) \int_{B_{t}\left(\xi_{0}\right)}\left|V(X u)-V\left(p_{0}\right)\right|^{2} d \xi \\
& \geq C(k, N, m, \lambda, M) \int_{B_{t}\left(\xi_{0}\right)}|V(X v)|^{2} d \xi,
\end{aligned}
$$

where we have applied Lemma 3(4) in the second inequality and Lemma 3 (6) for the final inequality.

The structure condition (H3) yields

$$
I \leq \int_{B_{s}\left(\xi_{0}\right)} K(\cdot)\left(1+\left|p_{0}\right|\right)^{m / 2}|v|^{\gamma}|X \varphi| d \xi .
$$

To obtain a suitable estimate for $I$, we need take the domain $B_{s}\left(\xi_{0}\right)$ into four parts: $B_{s}\left(\xi_{0}\right) \cap\{|v / s|>1\} \cap\{|X \varphi| \leq 1\}, B_{s}\left(\xi_{0}\right) \cap\{|v / s|>1\} \cap\{|X \varphi|>1\}$, $B_{s}\left(\xi_{0}\right) \cap\{|v / s| \leq 1\} \cap\{|X \varphi|>1\}, \quad B_{s}\left(\xi_{0}\right) \cap\{|v / s| \leq 1\} \cap\{|X \varphi| \leq 1\}$, and will use Young's inequality, (2.11) and (2.12), repeatedly.

CASE 1. On the part $B_{s}\left(\xi_{0}\right) \cap\{|v / s|>1\} \cap\{|X \varphi| \leq 1\}$, we see

$$
\begin{aligned}
& K(\cdot)\left(1+\left|p_{0}\right|\right)^{m / 2}\left|\frac{v}{s}\right|^{\gamma} s^{\gamma}|X \varphi| \\
\leq & C(\varepsilon)|X \varphi|^{2}+C(\varepsilon)\left|\frac{v}{s}\right|^{m}+C(\varepsilon)\left[K(\cdot)\left(1+\left|p_{0}\right|\right)^{m / 2} s^{\gamma}\right]^{2 m /(m-2 \gamma)} \\
\leq & C(\varepsilon)|V(X v)|^{2}+C(\varepsilon)\left|V\left(\frac{v}{s}\right)\right|^{2}+C(\varepsilon)\left[K(\cdot)\left(1+\left|p_{0}\right|\right)^{m / 2}\right]^{2 m /(m-2 \gamma)} s^{2 \gamma},
\end{aligned}
$$

where we have used the fact $s^{\gamma}<1$ and $2 m /(m-2 \gamma)>2$.

CASE 2. On the set $B_{s}\left(\xi_{0}\right) \cap\{|v / s|>1\} \cap\{|X \varphi|>1\}$, it holds

$$
\begin{aligned}
& K(\cdot)\left(1+\left|p_{0}\right|\right)^{m / 2}\left|\frac{v}{s}\right|^{\gamma} s^{\gamma}|X \varphi| \\
(4.16) \leq & C(\varepsilon)|X \varphi|^{m}+C(\varepsilon)\left|\frac{v}{s}\right|^{m}+C(\varepsilon)\left[K(\cdot)\left(1+\left|p_{0}\right|\right)^{m / 2} s^{\gamma}\right]^{m /(m-\gamma-1)} \\
\leq & C(\varepsilon)|V(X v)|^{2}+C(\varepsilon)\left|V\left(\frac{v}{s}\right)\right|^{2}+C(\varepsilon)\left[K(\cdot)\left(1+\left|p_{0}\right|\right)^{m / 2}\right]^{m /(m-\gamma-1)} s^{2 \gamma},
\end{aligned}
$$

where we have used $m /(m-\gamma-1)>2$. 
CASE 3. On the set $B_{s}\left(\xi_{0}\right) \cap\{|v / s| \leq 1\} \cap\{|X \varphi|>1\}$, observing $m /(m-1)>2$, one has

$$
\begin{aligned}
& K(\cdot)\left(1+\left|p_{0}\right|\right)^{m / 2}|v|^{\gamma}|X \varphi| \\
\leq & K(\cdot)\left(1+\left|p_{0}\right|\right)^{m / 2} s^{\gamma}|X \varphi| \\
\leq & \varepsilon|X \varphi|^{m}+C(\varepsilon)\left[K(\cdot)\left(1+\left|p_{0}\right|\right)^{m / 2} s^{\gamma}\right]^{m /(m-1)} \\
\leq & C(\varepsilon)|V(X v)|^{2}+C(\varepsilon)\left[K(\cdot)\left(1+\left|p_{0}\right|\right)^{m / 2}\right]^{m /(m-1)} s^{2 \gamma} .
\end{aligned}
$$

CASE 4. For $B_{s}\left(\xi_{0}\right) \cap\{|v / s| \leq 1\} \cap\{|X \varphi| \leq 1\}$, there is

$$
\begin{aligned}
& K(\cdot)\left(1+\left|p_{0}\right|\right)^{m / 2}|v|^{\gamma}|X \varphi| \\
\leq & K(\cdot)\left(1+\left|p_{0}\right|\right)^{m / 2} s^{\gamma}|X \varphi| \\
\leq & \varepsilon|X \varphi|^{2}+C(\varepsilon)\left[K(\cdot)\left(1+\left|p_{0}\right|\right)^{m / 2} s^{\gamma}\right]^{2} \\
\leq & C(\varepsilon)|V(X v)|^{2}+C(\varepsilon)\left[K(\cdot)\left(1+\left|p_{0}\right|\right)^{m / 2}\right]^{2} s^{2 \gamma} .
\end{aligned}
$$

Combining these estimations with (4.14), we get

$$
\begin{aligned}
I \leq & C(\varepsilon) \int_{B_{s}\left(\xi_{0}\right)}|V(X v)|^{2} d \xi+C(\varepsilon) \int_{B_{s}\left(\xi_{0}\right)}\left|V\left(\frac{v}{s}\right)\right|^{2} d \xi \\
& +C(\varepsilon)\left[K(\cdot)\left(1+\left|p_{0}\right|\right)^{m / 2}\right]^{\sigma}\left|B_{s}\left(\xi_{0}\right)\right|_{G} s^{2 \gamma},
\end{aligned}
$$

where $\sigma=\max \{2 m /(m-2 \gamma), m /(m-1-\gamma)\}>2$.

Similarly to $I$, it follows that

$$
\begin{aligned}
I I \leq & C(\varepsilon) \int_{B_{s}\left(\xi_{0}\right)}|V(X v)|^{2} d \xi+C(\varepsilon) \int_{B_{s}\left(\xi_{0}\right)}\left|V\left(\frac{v}{s}\right)\right|^{2} d \xi \\
& +C(\varepsilon)\left[K(\cdot)\left(1+\left|p_{0}\right|\right)^{m / 2+\gamma}\right]^{m /(m-1)}\left|B_{s}\left(\xi_{0}\right)\right|_{G} s^{2 \gamma}
\end{aligned}
$$

By (H1), Lemma 5 and (4.10), it holds

$$
\begin{aligned}
I I I & \leq C \int_{B_{s}\left(\xi_{0}\right)}\left[\int_{0}^{1}\left(1+\mid X u+\theta\left[(X u-X \phi)-\left.X u\right|^{2}\right)^{(m-2) / 2} d \theta\right]|X \phi||X \varphi| d \xi\right. \\
& \leq C \int_{B_{s}\left(\xi_{0}\right)}\left(1+|X u|^{2}+|X u-X \phi|^{2}\right)^{(m-2) / 2}|X \phi||X \varphi| d \xi \\
& \leq C \int_{B_{s}\left(\xi_{0}\right)}\left(1+|X u|^{2}+|X \phi|^{2}\right)^{(m-2) / 2}|X \phi||X \varphi| d \xi .
\end{aligned}
$$


Noting that $X \phi \subset B_{s} \backslash B_{t}$ and $-1 / 2<(m-2) / 2<0$, we split the domain $B_{s}\left(\xi_{0}\right)$ into four parts: $B_{s}\left(\xi_{0}\right) \cap\{|X \phi|>1\} \cap\{|X \varphi|>1\}, B_{s}\left(\xi_{0}\right) \cap\{|X \phi| \leq 1\} \cap$ $\{|X \varphi| \leq 1\}, \quad B_{s}\left(\xi_{0}\right) \cap\{|X \phi|>1\} \cap\{|X \varphi| \leq 1\}$, and $B_{s}\left(\xi_{0}\right) \cap\{|X \phi| \leq 1\} \cap$ $\{|X \varphi|>1\}$. Similarly to $I$, it follows that

$$
I I I \leq C\left(\int_{B_{s}\left(\xi_{0}\right) \backslash B_{t}\left(\xi_{0}\right)}\left|V\left(X u-p_{0}\right)\right|^{2} d \xi+\int_{B_{s}\left(\xi_{0}\right)}\left|V\left(\frac{v}{s-t}\right)\right|^{2} d \xi\right) .
$$

Using Hölder inequality, one has

$$
\begin{aligned}
I V & \leq C \int_{B_{s}\left(\xi_{0}\right)}\left(|X u|^{m}+|u|^{r}+1\right)^{1-\frac{1}{r}}|\varphi| d \xi \\
& \leq C\left[\int_{B_{s}\left(\xi_{0}\right)}\left(|X u|^{m}+|u|^{r}+1\right) d \xi\right]^{1-\frac{1}{r}}\left(\int_{B_{s}\left(\xi_{0}\right)}|\varphi|^{r} d \xi\right)^{\frac{1}{r}} .
\end{aligned}
$$

Analogously as $I$, we take the domain $B_{s}\left(\xi_{0}\right)$ into two parts.

CASE 1. For $B_{s}\left(\xi_{0}\right) \cap\{|X v|>1\}$, by Sobolev type inequality, Young's inequality and (2.12) it follows that

$$
\begin{aligned}
& {\left[\int_{B_{s}\left(\xi_{0}\right)}\left(|X u|^{m}+|u|^{r}+1\right) d \xi\right]^{1-\frac{1}{r}}\left(\int_{B_{s}\left(\xi_{0}\right)}|\varphi|^{r} d \xi\right)^{\frac{1}{r}} } \\
\leq & {\left[\int_{B_{s}\left(\xi_{0}\right)}\left(|X u|^{m}+|u|^{r}+1\right) d \xi\right]^{1-\frac{1}{r}}\left(\int_{B_{s}\left(\xi_{0}\right)}|X v|^{m} d \xi\right)^{\frac{1}{m}} } \\
\leq & C(\varepsilon)\left[\int_{B_{s}\left(\xi_{0}\right)}\left(|X u|^{m}+|u|^{r}+1\right) d \xi\right]^{\left(1-\frac{1}{r}\right) \frac{m}{m-1}}+C \varepsilon\left(\int_{B_{s}\left(\xi_{0}\right)}|X v|^{m} d \xi\right) \\
& {\left[\int_{B_{s}(\xi)}\left(|X u|^{m}+|u|^{r}+1\right) d \xi\right]^{\left(1-\frac{1}{r}\right) \frac{m}{m-1}}+C \varepsilon\left(\int_{B_{s}\left(\xi_{0}\right)}|V(X v)|^{2} d \xi\right) . }
\end{aligned}
$$

CASE 2. On the set $B_{s}\left(\xi_{0}\right) \cap\{|X v| \leq 1\}$, we have

$$
|X v|^{m} \leq|X v|^{2}+1,
$$


and then it follows that

$$
\begin{aligned}
& {\left[\int_{B_{s}\left(\xi_{0}\right)}\left(|X u|^{m}+|u|^{r}+1\right) d \xi\right]^{1-\frac{1}{r}}\left(\int_{B_{s}\left(\xi_{0}\right)}|\varphi|^{r} d \xi\right)^{\frac{1}{r}} } \\
\leq & C(\varepsilon)\left[\int_{B_{s}\left(\xi_{0}\right)}\left(|X u|^{m}+|u|^{r}+1\right) d \xi\right]^{\left(1-\frac{1}{r}\right) \frac{m}{m-1}} \\
+ & C \varepsilon\left(\int_{B_{s}\left(\xi_{0}\right)}|V(X v)|^{2} d \xi\right)+C \varepsilon\left|B_{s}\left(\xi_{0}\right)\right|_{G},
\end{aligned}
$$

where we have used (2.11).

Combining these estimates in $I V$, we have

$$
\begin{aligned}
& \text { (4.26) IV } \leq C \varepsilon\left(\int_{B_{s}\left(\xi_{0}\right)}|V(X v)|^{2} d \xi\right) \\
& +C(\varepsilon)\left[\int_{B_{s}\left(\xi_{0}\right)}\left(|X u|^{m}+|u|^{r}+1\right) d \xi\right]^{\left(1-\frac{1}{r}\right) \frac{m}{m-1}}+C \varepsilon\left|B_{s}\left(\xi_{0}\right)\right|_{G} .
\end{aligned}
$$

Substituting (4.13), (4.19), (4.20), (4.22) and (4.26) into (4.12), we finally arrive at

$$
\begin{aligned}
& C_{1} \int_{B_{t}\left(\xi_{0}\right)}|V(X v)|^{2} d \xi \\
\leq & C_{2} \int_{B_{s}\left(\xi_{0}\right) \backslash B_{t}\left(\xi_{0}\right)}\left(|V(X v)|^{2}+\left|V\left(\frac{v}{s-t}\right)\right|^{2}\right) d \xi \\
& +C_{3} \int\left(|V(X v)|^{2}+\left|V\left(\frac{v}{s-t}\right)\right|^{2}\right) d \xi+C_{4}\left[K(\cdot)\left(1+\left|p_{0}\right|^{m / 2}\right)\right]^{\sigma}\left|B_{s}\left(\xi_{0}\right)\right|_{G} s^{2 \gamma} \\
& +C_{s}\left(\xi_{0}\right) \\
& \left.\int_{B_{s}\left(\xi_{0}\right)}\left(|X u|^{m}+|u|^{r}+1\right) d \xi\right]^{\left(1-\frac{1}{r}\right) \frac{m}{m-1}}
\end{aligned}
$$

The proof is now completed by applying Lemma 4. 
REMARK 1. The function $v$ taken in the proof is different from that in [14], essentially.

\section{Proof of the main result}

In this section, we will complete the proof of Theorem 1 via four steps, and hence consider $u \in H W^{1, m}\left(\Omega, \mathbb{R}^{N}\right)(1<m<2)$ to be a weak solution of the system (1.1).

FIRst SteP. We claim that if $\rho \leq 1$ and $\varphi \in C_{0}^{\infty}\left(B_{\rho}\left(\xi_{0}\right),\left\{\mathbb{R}^{N}\right)\right.$ with $\sup |X \varphi| \leq 1$, then there exist some constants $C_{6}=C_{6}\left(m, M, C_{P}\right)>1$ $B_{\rho}\left(\xi_{0}\right)$ such that

$$
\begin{aligned}
& \left|B_{\rho}\left(\xi_{0}\right)\right|_{G}^{-1} \int_{B_{\rho}\left(\xi_{0}\right)} A_{i, p_{\beta}^{j}}^{\alpha}\left(\xi_{0}, u_{0}, p_{0}\right)\left(X u-p_{0}\right) X \varphi^{\alpha} d \xi \\
\leq & C_{6} \sup _{B_{\rho}\left(\xi_{0}\right)}|X \varphi|\left[\omega^{\frac{1}{2}}\left(\left|p_{0}\right|, \Phi^{\frac{1}{2}}\left(\xi_{0}, \rho, p_{0}\right)\right) \Phi^{\frac{1}{2}}\left(\xi_{0}, \rho, p_{0}\right)+\right. \\
+ & \left.\Phi\left(\xi_{0}, \rho, p_{0}\right)+\rho^{\gamma} F\left(\left|u_{0}\right|+\left|p_{0}\right|\right)\right],
\end{aligned}
$$

where we denote $F(\tau)=\left[K(\tau)(1+\tau)^{\sigma^{\prime}}\right]^{\frac{2 m}{m-\gamma}}$ with $\sigma^{\prime}=\max \left\{m, r-1, \frac{m}{2}+\gamma\right\}$.

Proof. Noting the fact

$$
\begin{aligned}
& \int_{B_{\rho}\left(\xi_{0}\right)}\left[\int_{0}^{1} A_{i, p_{\beta}^{j}}^{\alpha}\left(\xi_{0}, u_{0}, \theta X u+(1-\theta) p_{0}\right)\left(X u-p_{0}\right) d \theta\right] X \varphi^{\alpha} d \xi \\
= & \int_{B_{\rho}\left(\xi_{0}\right)}\left[\int_{0}^{1} \frac{d}{d \theta} A_{i}^{\alpha}\left(\xi_{0}, u_{0}, \theta X u+(1-\theta) p_{0}\right) d \theta\right] X \varphi^{\alpha} d \xi \\
= & \int_{B_{\rho}\left(\xi_{0}\right)}\left[A_{i}^{\alpha}\left(\xi_{0}, u_{0}, X u\right)-A_{i}^{\alpha}\left(\xi_{0}, u_{0}, p_{0}\right)\right] X \varphi^{\alpha} d \xi \\
= & \int_{B_{\rho}\left(\xi_{0}\right)}\left[A_{i}^{\alpha}\left(\xi_{0}, u_{0}, X u\right)-A_{i}^{\alpha}(\xi, u, X u)\right] X \varphi^{\alpha} d \xi+\int_{B_{\rho}\left(\xi_{0}\right)} B^{\alpha}(\xi, u, X u) \varphi^{\alpha} d \xi
\end{aligned}
$$


we have

$$
\begin{aligned}
\text { (5.3) } & \int_{B_{\rho}\left(\xi_{0}\right)} A_{i, p_{\beta}^{j}}^{\alpha}\left(\xi_{0}, u_{0}, p_{0}\right)\left(X u-p_{0}\right) X \varphi^{\alpha} d \xi \\
= & \int_{B_{\rho}\left(\xi_{0}\right)}\left[\int_{0}^{1} A_{i, p_{\beta}^{j}}^{\alpha}\left(\xi_{0}, u_{0}, p_{0}\right) d \theta\left(X u-p_{0}\right)\right] X \varphi^{\alpha} d \xi \\
\leq & \int_{B_{\rho}\left(\xi_{0}\right)}\left\{\int_{0}^{1}\left|A_{i, p_{\beta}^{j}}^{\alpha}\left(\xi_{0}, u_{0}, p_{0}\right)-A_{i, p_{\beta}^{j}}^{\alpha}\left(\xi_{0}, u_{0}, \theta X u+(1-\theta) p_{0}\right)\right|\left|X u-p_{0}\right| d \theta\right\} \sup _{B_{\rho}\left(\xi_{0}\right)}|X \varphi| d \xi \\
& +\int_{B_{\rho}\left(\xi_{0}\right)}\left|A_{i}^{\alpha}\left(\xi_{0}, u_{0}, X u\right)-A_{i}^{\alpha}\left(\xi, u_{0}+p_{0}\left(\xi^{1}-\xi_{0}^{1}\right), X u\right)\right| \sup _{B_{\rho}\left(\xi_{0}\right)}|X \varphi| d \xi \\
& +\int_{B_{\rho}\left(\xi_{0}\right)}\left|A_{i}^{\alpha}\left(\xi, u_{0}+p_{0}\left(\xi^{1}-\xi_{0}^{1}\right), X u\right)-A_{i}^{\alpha}(\xi, u, X u)\right| \sup _{B_{\rho}\left(\xi_{0}\right)}|X \varphi| d \xi \\
& +\int_{B_{\rho}\left(\xi_{0}\right)}\left(a|p|^{m\left(1-\frac{1}{r}\right)}+b|u|^{r-1}+c\right)|\varphi| d \xi \\
:= & I^{\prime}+I I^{\prime}+I I I^{\prime}+I V^{\prime} .
\end{aligned}
$$

Using (H1) and the estimate (1.6) yields (Note that $m-2<0$ )

$$
\begin{aligned}
I^{\prime} \leq & \int_{B_{\rho}\left(\xi_{0}\right)}\left[\int_{0}^{1}\left|A_{i, p_{\beta}^{j}}^{\alpha}\left(\xi_{0}, u_{0}, p_{0}\right)-A_{i, p_{\beta}^{j}}^{\alpha}\left(\xi_{0}, u_{0}, p_{0}+\theta\left(X u-p_{0}\right)\right)\right|^{\frac{1}{2}+\frac{1}{2}} d t\right]\left|X u-p_{0}\right| d \xi \\
\leq & C \int_{B_{\rho}\left(\xi_{0}\right)}\left\{\int_{0}^{1}\left[\left(1+\left|p_{0}\right|^{2}\right)^{\frac{m-2}{2}}+\left(1+\left|p_{0}+\theta\left(X u-p_{0}\right)\right|^{2}\right)^{\frac{m-2}{2}}\right]^{\frac{1}{2}}\right. \\
& \left.\times\left[\left(1+\left|p_{0}\right|^{2}+\left|p_{0}+\theta\left(X u-p_{0}\right)\right|^{2}\right)^{\frac{m-2}{2}} \omega\left(\left|p_{0}\right|,\left|\theta\left(X u-p_{0}\right)\right|\right)\right]^{\frac{1}{2}} d t\right\}\left|X u-p_{0}\right| d \xi \\
\leq & C \int_{B_{\rho}\left(\xi_{0}\right)}\left(1+\left|p_{0}\right|^{2}+|X u|^{2}\right)^{\frac{m-2}{4}} \omega^{\frac{1}{2}}\left(\left|p_{0}\right|,\left|X u-p_{0}\right|\right)\left|X u-p_{0}\right| d \xi \\
\leq & C \int_{B_{\rho}\left(\xi_{0}\right)}\left(1+\left|X u-p_{0}\right|^{\frac{m-2}{2}}\right)\left|X u-p_{0}\right| \omega^{\frac{1}{2}}\left(\left|p_{0}\right|,\left|X u-p_{0}\right|\right) d \xi .
\end{aligned}
$$

Let

(5.5) $\quad B_{1}=: B_{\rho}\left(\xi_{0}\right) \cap\left\{\left|X u-p_{0}\right| \leq 1\right\}, \quad B_{2}=: B_{\rho}\left(\xi_{0}\right) \cap\left\{\left|X u-p_{0}\right|>1\right\}$. 
Then it follows that by first Hölder's inequality and then Jensen's inequality

$$
\begin{aligned}
& I^{\prime} \leq C \int_{B_{1}}\left|X u-p_{0}\right| \omega^{\frac{1}{2}}\left(\left|p_{0}\right|,\left|X u-p_{0}\right|\right) d \xi \\
& +C \int_{B_{2}}\left|X u-p_{0}\right|^{\frac{m}{2}} \omega^{\frac{1}{2}}\left(\left|p_{0}\right|,\left|X u-p_{0}\right|\right) d \xi \\
& \leq C\left(\int_{B_{1}}\left|X u-p_{0}\right|^{2} d \xi\right)^{\frac{1}{2}}\left(\int_{B_{\rho}\left(\xi_{0}\right)} \omega\left(\left|p_{0}\right|,\left|X u-p_{0}\right|\right) d \xi\right)^{\frac{1}{2}} \\
& +C\left(\int_{B_{2}}\left|X u-p_{0}\right|^{m} d \xi\right)^{\frac{1}{2}}\left(\int_{B_{p}\left(\xi_{0}\right)} \omega\left(\left|p_{0}\right|,\left|X u-p_{0}\right|\right) d \xi\right)^{\frac{1}{2}} \\
& \leq C\left(\int_{B_{\rho}\left(\xi_{0}\right)}\left|V(X u)-V\left(p_{0}\right)\right|^{2} d \xi\right)^{\frac{1}{2}}\left(\int_{B_{p}\left(\xi_{0}\right)} \omega\left(\left|p_{0}\right|,\left|X u-p_{0}\right|\right) d \xi\right)^{\frac{1}{2}} \\
& +C\left(\int_{B_{\rho}\left(\xi_{0}\right)}\left|V(X u)-V\left(p_{0}\right)\right|^{2} d \xi\right)^{\frac{1}{2}}\left(\int_{B_{\rho}\left(\xi_{0}\right)} \omega\left(\left|p_{0}\right|,\left|X u-p_{0}\right|\right) d \xi\right)^{\frac{1}{2}} \\
& \leq 2 C\left|B_{\rho}\left(\xi_{0}\right)\right|_{G}\left(\left|B_{\rho}\left(\xi_{0}\right)\right|_{G}^{-1} \int_{B_{\rho}\left(\xi_{0}\right)}\left|V(X u)-V\left(p_{0}\right)\right|^{2} d \xi\right)^{\frac{1}{2}} \\
& +\left(\left|B_{\rho}\left(\xi_{0}\right)\right|_{G}^{-1} \int_{B_{\rho}\left(\xi_{0}\right)} \omega\left(\left|p_{0}\right|,\left|X u-p_{0}\right|\right) d \xi\right)^{\frac{1}{2}} \\
& \leq C\left|B_{\rho}\left(\xi_{0}\right)\right|_{G} \Phi^{\frac{1}{2}}\left(\xi_{0}, \rho, p_{0}\right) \omega^{\frac{1}{2}}\left(\left|p_{0}\right|,\left(\left|B_{\rho}\left(\xi_{0}\right)\right|_{G}^{-1} \int_{B_{1}}\left|X u-p_{0}\right|^{2} d \xi\right)^{\frac{1}{2}}\right. \\
& \left.+\left(\left|B_{\rho}\left(\xi_{0}\right)\right|_{G}^{-1} \int_{B_{2}}\left|X u-p_{0}\right|^{m} d \xi\right)^{\frac{1}{m}}\right)
\end{aligned}
$$




$$
\begin{aligned}
& \leq C\left|B_{\rho}\left(\xi_{0}\right)\right|_{G} \Phi^{\frac{1}{2}}\left(\xi_{0}, \rho, p_{0}\right) \omega^{\frac{1}{2}}\left(\left|p_{0}\right|,\left(\left|B_{\rho}\left(\xi_{0}\right)\right|_{G}^{-1} \int_{B_{\rho}\left(\xi_{0}\right)}\left|V(X u)-V\left(p_{0}\right)\right|^{2} d \xi\right)^{\frac{1}{2}}\right) \\
& =C\left|B_{\rho}\left(\xi_{0}\right)\right|_{G} \Phi^{\frac{1}{2}}\left(\xi_{0}, \rho, p_{0}\right) \omega^{\frac{1}{2}}\left(\left|p_{0}\right|, \Phi^{\frac{1}{2}}\left(\xi_{0}, \rho, p_{0}\right)\right)
\end{aligned}
$$

where we have used the estimates (2.11) and (2.12).

By employing (H3), the estimates (2.11) and (2.12), Young's inequality, and noting the fact that $K(\cdot)$ monotone nondecreasing and $K(\cdot)>1$ and that $\rho \leq 1$, we deduce

$$
\begin{aligned}
I I^{\prime} \leq & \int_{B_{\rho}\left(\xi_{0}\right)} K(\cdot) \rho^{\gamma}\left(1+\left|p_{0}\right|^{m}\right)^{\frac{\gamma}{m}}(1+|X u|)^{\frac{m}{2}} d \xi \\
\leq & \int_{B_{\rho}\left(\xi_{0}\right)} K(\cdot) \rho^{\gamma}\left(1+\left|p_{0}\right|\right)^{\gamma}\left[\left(1+\left|p_{0}\right|\right)^{\frac{m}{2}}+\left|X u-p_{0}\right|^{\frac{m}{2}}\right] d \xi \\
\leq & K(\cdot) \rho^{\gamma}\left(1+\left|p_{0}\right|\right)^{\gamma+\frac{m}{2}}\left|B_{\rho}\left(\xi_{0}\right)\right|_{G}+\int_{B_{1}+B_{2}} K(\cdot) \rho^{\gamma}\left(1+\left|p_{0}\right|\right)^{\gamma}\left|X u-p_{0}\right|^{\frac{m}{2}} d \xi \\
\leq & K(\cdot) \rho^{\gamma}\left(1+\left|p_{0}\right|\right)^{\gamma+\frac{m}{2}}\left|B_{\rho}\left(\xi_{0}\right)\right|_{G}+\left[K(\cdot) \rho^{\gamma}\left(1+\left|p_{0}\right|\right)^{\gamma}\right]^{2}\left|B_{\rho}\left(\xi_{0}\right)\right|_{G} \\
& +\left[K(\cdot) \rho^{\gamma}\left(1+\left|p_{0}\right|\right)^{\gamma}\right]^{\frac{4}{4-m}}\left|B_{\rho}\left(\xi_{0}\right)\right|_{G}+\int\left|X u-p_{0}\right|^{m} d \xi+\int\left|X u-p_{0}\right|^{2} d \xi \\
\leq & \left|B_{\rho}\left(\xi_{0}\right)\right|_{G} \Phi\left(\xi_{0}, \rho, p_{0}\right)+3\left[K(\cdot)\left(1+\left|p_{0}\right|\right)^{\gamma+\frac{m}{2}}\right]^{2}\left|B_{\rho}\left(\xi_{0}\right)\right|_{G} \rho^{\gamma} .
\end{aligned}
$$

Similarly to (4.19) to estimate $I I I^{\prime}$, the domain $B_{\rho}\left(\xi_{0}\right)$ is divided into four parts as previously mentioned. Then we obtain that by Lemma 3(6) and Lemma 3.1

$$
\begin{aligned}
I I I^{\prime} \leq & \int_{B_{\rho}\left(\xi_{0}\right)} K(\cdot)(1+|X u|)^{\frac{m}{2}}|v|^{\gamma} d \xi \\
\leq & C(\varepsilon, m, M)\left(\int_{B_{\rho}\left(\xi_{0}\right)}\left|V\left(\frac{v}{\rho}\right)\right|^{2} d \xi+\int_{B_{\rho}\left(\xi_{0}\right)}\left|V(X u)-V\left(p_{0}\right)\right|^{2} d \xi\right) \\
& +C(\varepsilon)\left[K(\cdot)\left(1+\left|p_{0}\right|\right)^{\frac{m}{2}}\right]^{\frac{2 m}{m-\gamma}} \rho^{\gamma}\left|B_{\rho}\left(\xi_{0}\right)\right|_{G} \\
\leq & C\left(\varepsilon, m, C_{P}, M\right) \Phi\left(\xi_{0}, \rho, p_{0}\right)\left|B_{\rho}\left(\xi_{0}\right)\right|_{G} \\
& +C(\varepsilon)\left[K(\cdot)\left(1+\left|p_{0}\right|\right)^{\frac{m}{2}}\right]^{\frac{2 m}{m-\gamma}} \rho^{\gamma}\left|B_{\rho}\left(\xi_{0}\right)\right|_{G} .
\end{aligned}
$$


With the help of the assumptions that $\sup _{B}|\varphi| \leq \rho \leq 1$, using Hölder's inequality, Sobolev type inequality and Young's inequality, we get

$$
\begin{aligned}
& I V^{\prime} \leq C \int_{B_{\rho}\left(\xi_{0}\right)}\left(|X u|^{m\left(1-\frac{1}{r}\right)}+|u|^{r-1}+1\right)|\varphi| d \xi \\
& \leq C \int_{B_{\rho}\left(\xi_{0}\right)}|X u|^{m\left(1-\frac{1}{r}\right)}|\varphi| d \xi+C \int_{B_{\rho}\left(\xi_{0}\right)}\left|u-u_{0}-p_{0}\left(\xi^{1}-\xi_{0}^{1}\right)\right|^{r-1}|\varphi| d \xi \\
& +C \rho\left|B_{\rho}\left(\xi_{0}\right)\right|\left[1+\left(\left|u_{0}\right|+\left|\rho p_{0}\right|\right)^{r-1}\right] \\
& \leq C\left(\int_{B_{\rho}\left(\xi_{0}\right)}|X u|^{m} d \xi\right)^{\left(1-\frac{1}{r}\right)}\left(\int_{B_{\rho}\left(\xi_{0}\right)}|\varphi|^{r} d \xi\right)^{\frac{1}{r}} \\
& +C \rho\left|B_{\rho}\left(\xi_{0}\right)\right|\left[1+\left(\left|u_{0}\right|+\left|p_{0}\right|\right)^{r-1}\right] \\
& +C\left(\int_{B_{\rho}\left(\xi_{0}\right)}\left|u-u_{0}-p_{0}\left(\xi^{1}-\xi_{0}^{1}\right)\right|^{r} d \xi\right)^{\left(1-\frac{1}{r}\right)}\left(\int_{B_{\rho}\left(\xi_{0}\right)}|\varphi|^{r} d \xi\right)^{\frac{1}{r}} \\
& \leq C\left(\int_{B_{\rho}\left(\xi_{0}\right)}\left(\left|X u-p_{0}\right|^{m}+\left|p_{0}\right|^{m}\right) d \xi\right)^{\left(1-\frac{1}{r}\right)}\left(\int_{B_{\rho}\left(\xi_{0}\right)}|\varphi|^{r} d \xi\right)^{\frac{1}{r}} \\
& +C\left(\int_{B_{\rho}\left(\xi_{0}\right)}\left|X u-p_{0}\right|^{m} d \xi\right)^{\frac{r-1}{m}}\left(\int_{B_{\rho}\left(\xi_{0}\right)}|\varphi|^{r} d \xi\right)^{\frac{1}{r}} \\
& +C \rho\left|B_{\rho}\left(\xi_{0}\right)\right|\left[1+\left(\left|u_{0}\right|+\left|p_{0}\right|\right)^{r-1}\right] \\
& \leq C\left(\int_{B_{\rho}\left(\xi_{0}\right)}\left|X u-p_{0}\right|^{m} d \xi\right)^{\frac{r-1}{r}}\left(\int_{B_{\rho}\left(\xi_{0}\right)}|\varphi|^{r} d \xi\right)^{\frac{1}{r}} \\
& +C\left(\int_{B_{\rho}\left(\xi_{0}\right)}\left|p_{0}\right|^{m} d \xi\right)^{\frac{r-1}{r}}\left(\int_{B_{\rho}\left(\xi_{0}\right)}|\varphi|^{r} d \xi\right)^{\frac{1}{r}}
\end{aligned}
$$




$$
\begin{aligned}
+ & C\left(\int_{B_{\rho}\left(\xi_{0}\right)}\left|X u-p_{0}\right|^{m} d \xi\right)^{\frac{r-1}{m}}\left(\int_{B_{\rho}\left(\xi_{0}\right)}|\varphi|^{r} d \xi\right)^{\frac{1}{r}} \\
+ & C \rho\left|B_{\rho}\left(\xi_{0}\right)\right|\left[1+\left(\left|u_{0}\right|+\left|p_{0}\right|\right)^{r-1}\right] \\
& \leq C\left(\int_{B_{\rho}\left(\xi_{0}\right)}\left|X u-p_{0}\right|^{m} d \xi\right)^{\frac{r-1}{r}}\left(\int_{B_{\rho}\left(\xi_{0}\right)}|\varphi|^{r} d \xi\right)^{\frac{1}{r}} \\
& +C \rho\left|B_{\rho}\left(\xi_{0}\right)\right|\left[1+\left(\left|u_{0}\right|+\left|p_{0}\right|\right)^{r-1}+\left|p_{0}\right|^{m\left(1-\frac{1}{r}\right)}\right] \\
\leq & C \int_{B_{\rho}\left(\xi_{0}\right)}\left|X u-p_{0}\right|^{m} d \xi+C \int_{B_{\rho}\left(\xi_{0}\right)}|\varphi|^{r} d \xi+C \rho\left|B_{\rho}\left(\xi_{0}\right)\right| \\
& \cdot\left[1+\left(\left|u_{0}\right|+\left|p_{0}\right|\right)^{r-1}+\left|p_{0}\right|^{m\left(1-\frac{1}{r}\right)}\right] \\
\leq & C \int_{B_{\rho}\left(\xi_{0}\right)}\left|X u-p_{0}\right|^{m} d \xi+C \rho^{r}\left|B_{\rho}\left(\xi_{0}\right)\right|+C \rho\left|B_{\rho}\left(\xi_{0}\right)\right| \\
& \cdot\left[1+\left(\left|u_{0}\right|+\left|p_{0}\right|\right)^{r-1}+\left|p_{0}\right|^{m\left(1-\frac{1}{r}\right)}\right] .
\end{aligned}
$$

On the case $B_{1}=: B_{\rho}\left(\xi_{0}\right) \cap\left\{\left|X u-p_{0}\right| \leq 1\right\}$, by (2.11) and Young's inequality, one gets

$$
\left|X u-p_{0}\right|^{m} \leq\left|X u-p_{0}\right|^{2}+1 \leq\left|V(X u)-V\left(p_{0}\right)\right|^{2}+1,
$$

and then

$$
\begin{aligned}
(5.9) \leq C \rho\left|B_{\rho}\left(\xi_{0}\right)\right|\left[\left(1+\left|u_{0}\right|+\left|p_{0}\right|\right)^{r-1}+(1+\right. & \left.\left.\left|u_{0}\right|+\left|p_{0}\right|\right)^{m}\right] \\
& +C\left|B_{\rho}\left(\xi_{0}\right)\right| \Phi\left(\xi_{0}, \rho, p_{0}\right) .
\end{aligned}
$$

On the other hand, on $B_{2}=: B_{\rho}\left(\xi_{0}\right) \cap\left\{\left|X u-p_{0}\right|>1\right\}$, by (2.12) and Young's inequality, one has

$$
\begin{aligned}
(5.9) \leq C \rho\left|B_{\rho}\left(\xi_{0}\right)\right|\left[\left(1+\left|u_{0}\right|+\left|p_{0}\right|\right)^{r-1}+(1+\right. & \left.\left.\left|u_{0}\right|+\left|p_{0}\right|\right)^{m}\right] \\
& +C\left|B_{\rho}\left(\xi_{0}\right)\right| \Phi\left(\xi_{0}, \rho, p_{0}\right) .
\end{aligned}
$$

Thus we infer that by combining these estimates and noting the definition of $F(\tau)$

$$
I V^{\prime} \leq C \Phi\left(\xi_{0}, \rho, p_{0}\right)\left|B_{\rho}\left(\xi_{0}\right)\right|_{G}+C F\left(\left|u_{0}\right|+\left|p_{0}\right|\right)\left|B_{\rho}\left(\xi_{0}\right)\right|_{G} \rho^{\gamma}
$$


Now, we can get (5.1) immediately by combining estimates $I^{\prime}, I I^{\prime}, I I I^{\prime}$ and $I V^{\prime}$ with (5.3).

We next establish an initial excess-improvement estimate, assuming that the excess $\Phi$ is initially small enough. Precisely,

SECOND STEP (Excess-improvement). Consider a weak solution $u \in H W^{1, m}\left(\Omega, \mathbb{R}^{N}\right)$ satisfying the condition of Theorem 1 . Then we claim that for a fixed $\gamma \in(0,1)$, there exist positive constants $C_{7}, C_{8}$ and $\delta$ satisfying conditions in Lemma 8 , and $\theta \in\left(0, \frac{1}{4}\right)$, such that the smallness conditions hold for some $\rho \in\left(0, \rho_{0}\right]$ :

$$
\begin{gathered}
\omega\left(\left|(X u)_{\xi_{0}, \rho}\right|, \Phi^{\frac{1}{2}}\left(\xi_{0}, \rho,(X u)_{\xi_{0}, \rho}\right)\right) \leq \frac{\delta^{2}}{16}, \\
\Phi\left(\xi_{0}, \rho,(X u)_{\xi_{0}, \rho}\right) \leq \min \left\{\frac{\delta^{2}}{16}, \frac{1}{8 C_{0}^{2} C_{6}^{2} C(m, M)}-4 \delta^{-2} H_{0}^{2} \rho^{2 \gamma}\right\},
\end{gathered}
$$

where $C(m, M)$ is a positive constant defined in Lemma 3 (6), and

$$
2 C_{7} \rho^{\gamma} H^{\frac{r-1}{m-1}}\left(1+\left|(X u)_{\xi_{0}, \rho}\right|\right) \leq \delta / 2 .
$$

Then three inequalities above imply the following growth condition

$$
\Phi\left(\xi_{0}, \theta \rho,(X u)_{\xi_{0}, \theta \rho}\right) \leq \theta^{2 \gamma}\left[\Phi\left(\xi_{0}, \rho,(X u)_{\xi_{0}, \rho}\right)+C_{8} \rho^{2 \gamma} H_{0}^{\frac{2(r-1)}{m-1}}\right],
$$

where $H_{0}=H\left(1+M_{1}\right)$.

Proof. Paying attention to properties of $\omega(s, t)$ in (1.6), we choose a suitable $t_{0}>0$ satisfying

$$
t_{0} \leq \min \left\{\frac{\delta^{2}}{16}, \frac{1}{8 C_{0}^{2} C_{6}^{2} C(m, M)}-4 \delta^{-2} H_{0}^{2} \rho^{2 \gamma}, \frac{M_{1}^{2} \theta^{Q}\left(1-\theta^{\gamma}\right)^{2}}{2}\right\},
$$

such that

$$
\omega\left(M_{1}, t_{0}\right) \leq \frac{\delta^{2}}{16}
$$

Moreover, let us pick $\rho_{0}>0$ such that $\rho_{0}^{2 \gamma}<\frac{t_{0}}{\left(4 C_{8}+C_{7}^{2}\right) H_{0}^{\frac{2(r-1)}{m-1}}}$ which ensures
that

$$
2 C_{7} \rho_{0}^{\gamma} H_{0}^{(r-1)} \leq \delta / 2, \quad 2 C_{8} \rho_{0}^{2 \gamma} H_{0}^{2(r-1)} \leq t_{0} / 2 .
$$


Taking $\rho \in\left(0, \rho_{0}\right]$ to correspond with

$$
\left|(X u)_{\xi_{0}, \rho}\right|<M_{1} / 2, \quad \Phi\left(\xi_{0}, \rho,(X u)_{\xi_{0}, \rho}\right)<t_{0} / 2,
$$

it follows that (5.13)-(5.15) are valid.

For simplicity, we will use the abbreviate $\Phi(\rho)=\Phi\left(\xi_{0}, \rho,(X u)_{\xi_{0}, \rho}\right)$ in the sequel. For $\varepsilon>0$ to be determined later, we take $\delta \in(0,1)$ and $\Upsilon \in[0,1]$ to be corresponding constant from the $\mathcal{A}$-harmonic approximation lemma (Lemma 8), and set

$$
w=u-\left(u_{\xi_{0}, \rho}-\Upsilon h_{\xi_{0}, 2 \theta \rho}\right)-(X u)_{\xi_{0}, \rho}\left(\xi^{1}-\xi_{0}^{1}\right),
$$

and

$$
\Upsilon=C(m, M) C_{6} \Gamma(\rho), \Gamma(\rho)=\sqrt{\Phi(\rho)+4 \delta^{-2} H_{0}^{2} \rho^{2 \gamma}} .
$$

Noting (5.13) and (5.14) yields

$$
\omega^{1 / 2}\left(\left|(X u)_{\xi_{0}, \rho}\right|, \Phi^{1 / 2}(\rho)\right)+\Phi^{1 / 2}(\rho) \leq \frac{\delta}{2},
$$

and by (5.1), we derive (note that the definition of $\Gamma(\rho)$ )

$$
\begin{aligned}
& \left.|| B_{\rho}\left(\xi_{0}\right)\right|_{G} ^{-1} \int_{B_{\rho}\left(\xi_{0}\right)}\left[A_{i, p_{\beta}^{j}}^{\alpha}\left(\xi_{0}, u_{\xi_{0}, \rho},(X u)_{\xi_{0}, \rho}\right) X w\right] X_{i} \varphi^{\alpha} d \xi \mid \\
\leq & \Upsilon \frac{\omega^{1 / 2}\left(\left|(X u)_{\xi_{0}, \rho}\right|, \Phi^{1 / 2}(\rho)\right) \Phi^{1 / 2}(\rho)+\Phi(\rho)+\rho^{\gamma} H\left(\left|(X u)_{\xi_{0}, \rho}\right|\right)}{C(m, M) \Gamma(\rho)} \sup _{B_{\rho}\left(\xi_{0}\right)}|X \varphi| \\
\leq & \Upsilon\left[\omega^{1 / 2}\left(\left|(X u)_{\xi_{0}, \rho}\right|, \Phi^{1 / 2}(\rho)\right)+\Phi^{1 / 2}(\rho)+\frac{\delta}{2}\right] \sup _{B_{\rho}\left(\xi_{0}\right)}|X \varphi| \\
\leq & \Upsilon \delta \sup _{B_{\rho}\left(\xi_{0}\right)}|X \varphi| .
\end{aligned}
$$

Then from the definition of $\Upsilon$, Lemma $3(6)$ and (2.10), we have

$$
\begin{aligned}
&\left|B_{\rho}\left(\xi_{0}\right)\right|_{G}^{-1} \int_{B_{\rho}\left(\xi_{0}\right)}|W(X w)|^{2} d \xi \\
& \leq\left|B_{\rho}\left(\xi_{0}\right)\right|_{G}^{-1} \int_{B_{\rho}\left(\xi_{0}\right)}|V(X w)|^{2} d \xi \leq C(m, M) \Phi(\rho) \leq \Upsilon^{2} .
\end{aligned}
$$

Inequalities (5.22) and (5.23) fulfill the conditions of $\mathcal{A}$-harmonic approximation lemma, which ensures us to find an $\mathcal{A}$-harmonic function 
$h \in W^{1, m}\left(B_{\rho}\left(\xi_{0}\right), \mathbb{R}^{N}\right)$ such that

(5.24) $\left|B_{\rho}\left(\xi_{0}\right)\right|_{G}^{-1} \int_{B_{\rho}\left(\xi_{0}\right)}|W(X h)|^{2} d \xi \leq 1,\left|B_{\rho}\left(\xi_{0}\right)\right|_{G}^{-1} \int_{B_{\rho}\left(\xi_{0}\right)}\left|W\left(\frac{w-\Upsilon h}{\rho}\right)\right|^{2} d \xi \leq \Upsilon^{2} \varepsilon$.

With the help of Lemma 3(3) and (6), we have

$$
\begin{aligned}
\Phi^{2}(\theta \rho)= & \left|B_{\theta \rho}\left(\xi_{0}\right)\right|_{G}^{-1} \int_{B_{\theta \rho}\left(\xi_{0}\right)}\left|V(X u)-V\left((X u)_{\xi_{0, \theta_{\rho}}}\right)\right|^{2} d \xi \\
\leq & C\left|B_{\theta \rho}\left(\xi_{0}\right)\right|_{G}^{-1} \int_{B_{\theta \rho}\left(\xi_{0}\right)}\left|V\left(X u-(X u)_{\xi_{0, \theta_{\rho}}}\right)\right|^{2} d \xi \\
\leq & C\left|B_{\theta \rho}\left(\xi_{0}\right)\right|_{G}^{-1} \int_{B_{\theta \rho}\left(\xi_{0}\right)}\left|V\left(X u-(X u)_{\xi_{0, \theta_{\rho}}}-\Upsilon(X h)_{\left(\xi_{0}, 2 \theta \rho\right)}\right)\right|^{2} d \xi \\
& +C\left|V\left((X u)_{x_{0, \theta_{\rho}}}-(X u)_{\xi_{0, \rho}}-\Upsilon(X h)_{\left(\xi_{0}, 2 \theta \rho\right)}\right)\right|^{2},
\end{aligned}
$$

where the constant $C$ depends only on $m, k$ and $N$.

Next, we proceed to estimate the right-hand side of (5.25). Decomposing $B_{\theta \rho}\left(\xi_{0}\right)$ into the set with

$\left|X u-(X u)_{\xi_{0}, \rho}-\Upsilon(X h)_{\left(\xi_{0}, 2 \theta \rho\right)}\right| \leq 1$ and that with $\left|X u-(X u)_{\xi_{0}, \rho}-\Upsilon(X h)_{\left(\xi_{0}, 2 \theta \rho\right)}\right|>1$,

then by Lemma 3 (1) and Hölder inequality, we obtain

$$
\begin{aligned}
& \left|(X u)_{\xi_{0, \rho} \rho}-(X u)_{\xi_{0}, \rho}-\Upsilon(X h)_{\left(\xi_{0}, 2 \theta \rho\right)}\right| \\
= & \left.|| B_{\theta \rho}\left(\xi_{0}\right)\right|_{G} ^{-1} \int_{B_{\theta \rho}\left(\xi_{0}\right)}\left[X u-(X u)_{\xi_{0}, \rho}-\Upsilon(X h)_{\left(\xi_{0}, 2 \theta \rho\right)}\right] d \xi \mid \\
\leq & \left|B_{\theta \rho}\left(\xi_{0}\right)\right|_{G}^{-1} \int_{B_{\theta \rho}\left(\xi_{0}\right)}\left|X u-(X u)_{\xi_{0, \rho}}-\Upsilon(X h)_{\left(\xi_{0}, 2 \theta \rho\right)}\right| d \xi \\
\leq & \sqrt{2}\left(\Xi^{1 / 2}+\Xi^{1 / m}\right),
\end{aligned}
$$

where we have abbreviated

$$
\Xi=:\left|B_{\theta \rho}\left(\xi_{0}\right)\right|_{G}^{-1} \int_{B_{\theta \rho}\left(\xi_{0}\right)}\left|V\left(X u-(X u)_{\xi_{0}, \rho}-\Upsilon(X h)_{\left(\xi_{0}, 2 \theta \rho\right)}\right)\right|^{2} d \xi .
$$


Now, since $|V(\varsigma)|=V(|\varsigma|)$, and $V(\varsigma)$ is monotone increasing in $\varsigma$, it follows that from (5.25), and Lemma 3(1) and (2)

$$
\Phi^{2}(\theta \rho) \leq C\left(\Xi+V^{2}\left(\Xi^{1 / 2}+\Xi^{1 / m}\right)\right) \leq C\left(\Xi+\Xi^{2 / m}\right),
$$

where the constant $C$ depends only on $m, Q$ and $N$. Then it remains for us to estimate $\Xi$. By considering the cases $|X h| \leq 1$ and $|X h|>1$, separately and keeping in mind (5.24), we get

$$
\left|B_{\rho}\left(\xi_{0}\right)\right|_{G}^{-1} \int_{B_{\rho}\left(\xi_{0}\right)}|X h| d \xi \leq 2 \sqrt{2},
$$

where we have used Lemma 3(1).

Noting the assumption $\left|(X u)_{\xi_{0}, \rho}\right| \leq M_{1}, \theta \in(0,1 / 4),(5.14)$ and Lemma 7 , we deduce

$$
\begin{aligned}
\left|(X u)_{\xi_{0}, \rho}\right|+\Upsilon\left|(X h)_{\left(\xi_{0}, 2 \theta \rho\right)}\right| & \leq M_{1}+\Upsilon \sup _{B_{\rho / 2}\left(\xi_{0}\right)}|X h| \\
& \leq M_{1}+\Upsilon C_{0}\left|B_{\rho}\left(\xi_{0}\right)\right|_{G}^{-1} \int_{B_{\rho}\left(\xi_{0}\right)}|X h| d \xi \\
& \leq M_{1}+2 \sqrt{2} \Upsilon C_{0} \\
& \leq M_{1}+1 .
\end{aligned}
$$

Caccioppoli type inequality applied on $B_{\theta \rho}\left(\xi_{0}\right)$ yields

$$
\begin{aligned}
\Xi \leq C_{c} & {\left[\left|B_{2 \theta \rho}\left(\xi_{0}\right)\right|_{G}^{-1} \int_{B_{2 \theta_{\rho}\left(\xi_{0}\right)}}\right.} \\
& \left.\cdot\left|V\left(\frac{u-u_{\xi_{0}, \rho}-\left((X u)_{\xi_{0}, \rho}+\Upsilon(X h)_{\left(\xi_{0}, 2 \theta \rho\right)}\right)\left(\xi^{1}-\xi_{0}^{1}\right)}{2 \theta \rho}\right)\right|^{2} d \xi+U\right]
\end{aligned}
$$

where

$$
\begin{aligned}
U= & {\left[K\left(\left|u_{\xi_{0}, \rho}\right|+\left|(X u)_{\xi_{0}, \rho}+\Upsilon(X h)_{\left(\xi_{0}, 2 \theta \rho\right)}\right|\right)\left(1+\left|(X u)_{\xi_{0}, \rho}+\Upsilon(X h)_{\left(\xi_{0}, 2 \theta \rho\right)}\right|\right)^{m / 2}\right]^{\sigma}(2 \theta \rho)^{2 \gamma} } \\
& +\left|B_{\rho}\left(\xi_{0}\right)\right|_{G}^{-1}\left[\int_{B_{\rho}\left(\xi_{0}\right)}\left(|X u|^{m}+|u|^{r}+1\right) d \xi\right]^{\frac{m}{m-1}\left(1-\frac{1}{r}\right)} .
\end{aligned}
$$


By lemma 3(3), one gets

$$
\int_{B_{2 \theta \rho}\left(\xi_{0}\right)}\left|V\left(\frac{u-u_{\xi_{0}, \rho}-\left((X u)_{\xi_{0}, \rho}+\Upsilon(X h)_{\left(\xi_{0}, 2 \theta \rho\right)}\right)\left(\xi^{1}-\xi_{0}^{1}\right)}{2 \theta \rho}\right)\right|^{2} d \xi
$$$$
\leq \int_{B_{2 \theta \rho}\left(\xi_{0}\right)}\left|V\left(\begin{array}{c}
\frac{u-\left(u \xi_{0}, \rho\right.}{\left.-\Upsilon h_{\xi_{0}, 2 \theta \rho}\right)-(X u)_{\xi_{0}, \rho}\left(\xi^{1}-\xi_{0}^{1}\right)-\Upsilon h(\xi)} \\
2 \theta \rho \\
+\frac{\Upsilon h(\xi)-\Upsilon h_{\xi_{0}, 2 \theta \rho}-\Upsilon(X h)_{\left(\xi_{0}, 2 \theta \rho\right)}\left(\xi^{1}-\xi_{0}^{1}\right)}{2 \theta \rho}
\end{array}\right)\right| d \xi
$$

$\leq C\left[\int_{B_{2 \theta \rho}\left(\xi_{0}\right)}\left(\left|V\left(\frac{w-\Upsilon h(\xi)}{2 \theta \rho}\right)\right|^{2}+\left|V\left(\Upsilon \frac{h(\xi)-h_{\xi_{0}, 2 \theta \rho}-(X h)_{\left(\xi_{0}, 2 \theta \rho\right)}\left(\xi^{1}-\xi_{0}^{1}\right)}{2 \theta \rho}\right)\right|^{2}\right) d \xi\right]$.

To estimate the right-hand side we employ (2.10), Lemma 3(2) (note that $\left.\frac{1}{2 \theta} \geq 1\right)$ and (5.24) to infer

$$
\begin{aligned}
& \left|B_{2 \theta \rho}\left(\xi_{0}\right)\right|_{G}^{-1} \int_{B_{2 \theta \rho}\left(\xi_{0}\right)}\left|V\left(\frac{w-\Upsilon h(\xi)}{2 \theta \rho}\right)\right|^{2} d \xi \\
\leq & C\left|B_{2 \theta \rho}\left(\xi_{0}\right)\right|_{G}^{-1} \int_{B_{2 \theta_{\rho}}\left(\xi_{0}\right)}\left|W\left(\frac{w-\Upsilon h(\xi)}{2 \theta \rho}\right)\right|^{2} d \xi \\
\leq & C(2 \theta)^{-Q}\left|B_{\rho}\left(\xi_{0}\right)\right|_{G}^{-1} \int_{B_{\rho}\left(\xi_{0}\right)}\left|W\left(\frac{w-\Upsilon h(\xi)}{2 \theta \rho}\right)\right|^{2} d \xi \\
\leq & C(2 \theta)^{-Q-2}\left|B_{\rho}\left(\xi_{0}\right)\right|_{G}^{-1} \int_{B_{\rho}\left(\xi_{0}\right)}\left|W\left(\frac{w-\Upsilon h(\xi)}{\rho}\right)\right|^{2} d \xi \\
\leq & C(2 \theta)^{-Q-2} \Upsilon^{2} \varepsilon .
\end{aligned}
$$

Using Lemma 3, lemma 7, (2.10) and (5.24) leads to

$$
\begin{aligned}
& \left|B_{2 \theta \rho}\left(\xi_{0}\right)\right|_{G}^{-1} \int_{B_{2 \theta \rho}\left(\xi_{0}\right)}\left|V\left(\Upsilon \frac{h(\xi)-h_{\xi_{0}, 2 \theta \rho}-(X h)_{\left(\xi_{0}, 2 \theta \rho\right)}\left(\xi^{1}-\xi_{0}^{1}\right)}{2 \theta \rho}\right)\right|^{2} d \xi \\
\leq & \Upsilon^{2}\left|B_{2 \theta \rho}\left(\xi_{0}\right)\right|_{G}^{-1} \int_{B_{2 \theta \rho}\left(\xi_{0}\right)}\left|V\left(\frac{h(\xi)-h_{\xi_{0}, 2 \theta \rho}-(X h)_{\left(\xi_{0}, 2 \theta \rho\right)}\left(\xi^{1}-\xi_{0}^{1}\right)}{2 \theta \rho}\right)\right|^{2} d \xi
\end{aligned}
$$




$$
\begin{aligned}
& \leq C(2 \theta \rho)^{2} \Upsilon^{2}\left|B_{2 \theta \rho}\left(\xi_{0}\right)\right|_{G}^{-1} \int_{B_{2 \theta \rho}\left(\xi_{0}\right)}\left|V\left(X^{2} h\right)\right|^{2} d \xi \\
& \leq C(2 \theta \rho)^{2} \Upsilon^{2} \sup _{B_{\rho / 2}\left(\xi_{0}\right)}\left|X^{2} h\right|^{2} \\
& \leq C(2 \theta)^{2} \Upsilon^{2}\left|B_{\rho}\left(\xi_{0}\right)\right|_{G}^{-1} \int_{B_{\rho}\left(\xi_{0}\right)}|(X h)|^{2} d \xi \\
& \leq C(2 \theta)^{2} \Upsilon^{2}\left|B_{\rho}\left(\xi_{0}\right)\right|_{G}^{-1} \int_{B_{\rho}\left(\xi_{0}\right)}|W(X h)|^{2} d \xi \\
& \leq C \theta^{2} \Upsilon^{2} .
\end{aligned}
$$

Using the smallness condition $\Upsilon(X h)_{\left(\xi_{0}, 2 \theta \rho\right)} \leq 2 \sqrt{2} C_{0} \Upsilon \leq 1$ and the definition of $H(\cdot)$ yields

$$
\begin{aligned}
& {\left[K\left(\left|u_{\xi_{0}, \rho}\right|+\left|(X u)_{\xi_{0}, \rho}+\Upsilon(X h)_{\left(\xi_{0}, 2 \theta \rho\right)}\right|\right)\left(1+\left|(X u)_{\xi_{0}, \rho}+\Upsilon(X h)_{\left(\xi_{0}, 2 \theta \rho\right)}\right|\right)^{m / 2}\right]^{\sigma}(2 \theta \rho)^{2 \gamma}} \\
& \leq\left[K\left(M+\left|(X u)_{\xi_{0}, \rho}\right|+1\right)\left(2+\left|(X u)_{\xi_{0}, \rho}\right|\right)^{m / 2}\right]^{\sigma}(2 \theta \rho)^{2 \gamma} \\
& \leq H\left(\left|(X u)_{\xi_{0}, \rho}\right|+1\right)(2 \theta \rho)^{2 \gamma}
\end{aligned}
$$

Applying Sobolev type inequality, and noting $\frac{Q m}{m-1}\left(1-\frac{1}{r}\right)=$ $Q+\frac{Q}{m-1}\left(1-\frac{m}{r}\right)>Q+2, \frac{Q(r-1)}{m-1}=Q+Q\left(\frac{r-1}{m-1}-1\right)>Q+2, \theta \rho<1$ and $\gamma \in(0,1)$, we have

$$
\begin{aligned}
& \text { (5.33) }\left[\int_{B_{2 \theta_{\rho}}\left(\xi_{0}\right)}\left(|X u|^{m}+|u|^{r}+1\right) d \xi\right]^{\frac{m}{m-1}\left(1-\frac{1}{r}\right)} \\
& \leq\left[2^{m-1} \int_{B_{2 \theta \rho}\left(\xi_{0}\right)}\left(\left|X u-p_{0}\right|^{m}+\left|p_{0}\right|^{m}\right) d \xi+\int_{B_{2 \theta_{\rho}}\left(\xi_{0}\right)}|u|^{r} d \xi+\omega_{G}\left(\xi_{0}\right)(2 \theta \rho)^{Q}\right]^{\frac{m}{m-1}\left(1-\frac{1}{r}\right)} \\
& \leq 2^{m-1}\left\{\left(\int_{B_{2 \theta \rho}\left(\xi_{0}\right)}\left|X u-p_{0}\right|^{m} d \xi\right)^{\frac{m}{m-1}\left(1-\frac{1}{r}\right)}\right.
\end{aligned}
$$




$$
\begin{aligned}
& \left.+\left(1+\left|p_{0}\right|^{\frac{m^{2}}{m-1}\left(1-\frac{1}{r}\right)}\right)\left[\omega_{G}\left(\xi_{0}\right)(2 \theta \rho)^{Q}\right]^{\frac{m}{m-1}\left(1-\frac{1}{r}\right)}+\left(\int_{B_{2 \theta \rho}\left(\xi_{0}\right)}|X u|^{m} d \xi\right)^{\frac{r-1}{m-1}}\right\} \\
& \leq 2^{2(m-1)}\left\{\left(\int_{B_{2 \theta_{\rho}}\left(\xi_{0}\right)}\left|X u-p_{0}\right|^{m} d \xi\right)^{\frac{m}{m-1}\left(1-\frac{1}{r}\right)}+\left[\omega_{G}(2 \theta \rho)^{Q}\right]^{\frac{m}{m-1}\left(1-\frac{1}{r}\right)}\left(1+\left|p_{0}\right|^{\frac{m^{2}}{m-1}\left(1-\frac{1}{r}\right)}\right)\right. \\
& \left.+\left(\int_{B_{2 \theta \rho}\left(\xi_{0}\right)}\left|X u-p_{0}\right|^{m} d \xi\right)^{\frac{r-1}{m-1}}+\left|p_{0}\right|^{\frac{m(r-1)}{m-1}}\left[\omega_{G}(2 \theta \rho)^{Q}\right]^{\frac{r-1}{m-1}}\right\} \\
& \leq C\left\{\left(\int_{B_{2 \theta \rho}\left(\xi_{0}\right)}\left|X u-p_{0}\right|^{m} d \xi\right)^{\frac{m}{m-1}\left(1-\frac{1}{r}\right)}+\left(\int_{B_{2 \theta \rho}\left(\xi_{0}\right)}\left|X u-p_{0}\right|^{m} d \xi\right)^{\frac{r-1}{m-1}}\right. \\
& \left.+\omega_{G}\left[\left(1+\left|p_{0}\right|\right)^{m}\right]^{\frac{m}{m-1}\left(1-\frac{1}{r}\right)}\left[\left(1+\left|p_{0}\right|\right)^{m}\right]^{\frac{r-1}{m-1}}(\theta \rho)^{Q+2 \gamma}\right\}
\end{aligned}
$$

Assume that:

$$
(2 \theta)^{-Q} \Phi\left(\xi_{0}, \rho, p_{0}\right) \leq 1
$$

Then on the case $\left|X u-p_{0}\right| \leq 1$, from Hölder inequality, (2.11) and the definition of $H$, it follows that

$$
(5.33) \leq C \omega_{G}(2 \theta \rho)^{Q+2 \gamma} H^{\frac{2(r-1)}{m-1}}(1+M),
$$

where we have used the fact that $\frac{r-1}{m-1}+\frac{m}{m-1}\left(1-\frac{1}{r}\right)=$ $\frac{r-1}{m-1}\left(1+\frac{m}{r}\right) \leq \frac{2(r-1)}{m-1}$.

While on the case $\left|X u-p_{0}\right|>1$, using (2.12) together with the definition of $H$, we derive that

$$
(5.33) \leq C \omega_{G}(2 \theta \rho)^{Q+2 \gamma} H^{\frac{2(r-1)}{m-1}}(1+M) .
$$

Combining all the above estimates with (5.29), and let $\varepsilon=\theta^{Q+4}$, we arrive at

$$
\Xi \leq C_{7}\left[\theta^{2} \Upsilon^{2}+H^{\frac{2(r-1)}{m-1}}(1+M)(2 \theta \rho)^{2 \gamma}\right],
$$

where $C_{7}$ depends only on $Q, N, m, M, \lambda$ and $C_{P}$. For given $\gamma \in(0,1)$, 
Choosing $\theta \in\left(0, \frac{1}{4}\right)$ suitable such that $C_{7} \theta^{2} \leq \theta^{2 \gamma}$, we easily find (note that the definition of $\Upsilon$ )

$$
\Phi(\theta \rho) \leq \theta^{2 \gamma}\left[\Phi(\rho)+C_{8} H^{\frac{2(r-1)}{m-1}}(1+M) \rho^{2 \gamma}\right] .
$$

where the constant $C_{8}$ has the same dependencies as $C_{7}$.

THIRD STEP. We claim that for every $n \in \mathbb{N}$,

$$
\left|(X u)_{\xi_{0}, \theta^{n} \rho}\right|<M_{1}, \quad \Phi\left(\theta^{n} \rho\right)<t_{0} .
$$

It allows us to make iteration from the assertion in Second step and have

$$
\Phi\left(\theta^{n+1} \rho\right) \leq \theta^{2 \gamma}\left(\frac{1}{2} \Phi\left(\theta^{n} \rho\right)+C_{8}\left(\theta^{n} \rho\right)^{2 \gamma} H_{0}^{2}\right) .
$$

Proof. Like in the proof of the Third step in [18], we obtain the conclusion, and we omit the details here.

Fourth step. We claim that $u \in \Gamma^{1, \gamma}\left(\Omega_{0},\left\{\mathbb{R}^{N}\right)\right.$, where $\Omega_{0} \subset \Omega$ is an open subset such that Haar meas $\left(\Omega \backslash \Omega_{0}\right)=0$.

Proof. We have from Third step that

$$
\Phi\left(\theta^{n} \rho\right) \leq \theta^{2 n \gamma} t_{0}, \quad \text { for } n \in \mathbb{N} .
$$

Since for every $\rho \in\left(0, \rho_{0}\right]$, there exists a positive constant $n$, such that

$$
\theta^{n+1} \rho_{0}<\rho \leq \theta^{n} \rho_{0},
$$

we have that for given $\theta$ and $\rho_{0}$,

$$
\begin{aligned}
\Phi\left(\xi_{0}, \rho\right) & =\left|B_{\rho}\left(\xi_{0}\right)\right|_{G}^{-1} \int_{B_{\rho}\left(\xi_{0}\right)}\left|V\left(X u-p_{0}\right)\right|^{2} d \xi \\
& \leq\left|B_{\theta^{n+1} \rho_{0}}\left(\xi_{0}\right)\right|_{G}^{-1} \int_{B_{\theta^{n} \rho_{0}}\left(\xi_{0}\right)}\left|V\left(X u-p_{0}\right)\right|^{2} d \xi \\
& \leq C\left|B_{\theta^{n} \rho_{0}}\left(\xi_{0}\right)\right|_{G}^{-1} \int_{B_{\theta^{n} \rho_{0}}\left(\xi_{0}\right)}\left|V\left(X u-p_{0}\right)\right|^{2} d \xi \\
& =C \Phi\left(\theta^{n} \rho_{0}\right) \leq C \theta^{2 n \gamma} t_{0} \leq C\left(\theta^{-1} \frac{\rho}{\rho_{0}}\right)^{2 \gamma} \leq C \rho^{2 \gamma}
\end{aligned}
$$


Noting that (5.20) is still valid for any $\xi$ sufficiently close to $\xi_{0}$, there exists a ball $B_{r}\left(\xi_{0}\right)$ centered at $\xi_{0}$, such that for any $\xi \in B_{r}\left(\xi_{0}\right)$,

$$
\Phi(\xi, \rho) \leq C \rho^{2 \gamma} .
$$

Therefore we infer that $V(X u) \in \mathcal{L}^{2, \mu}\left(B_{\rho}\left(\xi_{0}\right), \mathbb{R}^{N}\right)$ with $\mu=Q+2 \gamma$. Using Lemma 2 and Lemma 3 implies $X u \in \Gamma^{\gamma}\left(B_{\rho}\left(\xi_{0}\right), \mathbb{R}^{N}\right)$.

\section{REFERENCES}

[1] E. De GioRgi, Un esempio di estremali discontinue per un problema variazionale di tipo ellitico. Boll. Unione Mat. Italiana 4 (1968), pp. 135-137.

[2] M. Giaquinta, Multiple Integrals in the Calculus of Variations and Nonlinear Elliptic Systems. Princeton Univ. Press, Princeton, 1983.

[3] M. Giaquinta, Introduction to regularity theory for nonlinear elliptic systems. Birkhäuser, Berlin, 1993.

[4] Y. CHEN - L. Wu, Second order elliptic equations and elliptic systems. Science Press, Beijing, 2003.

[5] M. Giaquinta - G. Modica, Almost-everywhere regularity results for solutions of non linear elliptic systems. Manuscripta Math. 28 (1979), pp. 109-158,

[6] E. Giusti - M. Miranda, Sulla regolarità delle soluzioni deboli di una classe di sistemi ellittici quasilineari. Arch. Rat. Mech. Anal. 31 (1968), pp. 173-184.

[7] F. DuZAar - J. F. Grotowski, Partial regularity for nonlinear elliptic systems: The method of A-harmonic approximation, Manuscripta Math. 103 (2000), pp. 267-298.

[8] L. Simon, Lectures on Geometric Measure Theory. Australian National University Press, Canberra, 1983.

[9] F. DuzaAR - J. F. GRotowski - M. Kronz, Regularity of almost minimizers of quasi-convex variational integrals with subquadratic growth. Annali Mat. Pura Appl. (4) 184 (2005), pp. 421-448.

[10] F. DUZAAR - G. Mingione, The p-harmonic approximation and the regularity of p-harmonic maps. Calc. Var. Partial Differential Equations 20 (2004), pp. $235-256$.

[11] F. DuZAar - G. Mingione, Regularity for degenerate elliptic problems via p-harmonic approximation. Ann. Inst. H. Poincaré Anal. Non Linèaire 21 (2004), pp. 735-766.

[12] M. Carozza - N. Fusco - G. Mingione, Partial regularity of minimizers of quasiconvex integrals with subquadratic growth, Annali Mat. Pura Appl. (4) 175 (1998), pp. 141-164.

[13] S. CHEN - Z. TAN, The method of A-harmonic approximation and optimal interior partial regularity for nonlinear elliptic systems under the controllable growth condition. J. Math. Anal. Appl. 335 (2007), pp. 20-42.

[14] S. CHEN - Z. TAN, Optimal interior partial regularity for nonlinear elliptic systems. Discrete Contin. Dyn. Syst. 27 (2010), pp. 981-993.

[15] L. Capogna - N. Garofalo, Regularity of minimizers of the calculus of variations in Carnot groups via hypoellipticity of systems of Hörmander type. J. European Math. Society 5 (2003), pp. 1-40. 
[16] E. SHores, Hypoellipticity for linear degenerate elliptic systems in Carnot groups and applications, arXiv:math/0502569, pp. 27.

[17] A. FöGLein, Partial regularity results for subelliptic systems in the Heisenberg group, Calc. Var. Partial Differential Equations 32 (2008), pp. 25-51.

[18] J. WANG - P. NIU, Optimal partial regularity for weak solutions of nonlinear sub-elliptic systems in Carnot groups. Nonlinear Analysis 72 (2010), pp. 41624187.

[19] L. Capogna - D. Danielli - N. Garofalo, An embedding theorem and the Harnak inequality for nonlinear subelliptic equations. Comm. Partial Differential Equations 18 (1993), pp. 1765-1794.

[20] G. Lu, Embedding theorems into Lipschitz and BMO spaces and applications to quasilinear subelliptic differential equations. Publ. Mat. 40 (1996), pp. 301-329.

[21] C. XU, Regularity for quasi-linear second order subelliptic equations. Comm. Pure Appl. Math. 45 (1992), pp. 77-96.

[22] L. CAPOGNA, Regularity of quasi-linear equations in the Heisenberg group. Comm. Pure Appl. Math. 50 (1997), pp. 867-889.

[23] L. CaPoGNA, Regularity for quasilinear equation and 1-quasiconformal maps in Carnot groups. Math. Ann. 313 (1999), pp. 263-295.

[24] S. MARchI, $C^{1, \alpha}$ local regularity for the solutions of the $p$-Laplacian on the Heisenberg group for $2<p<1+\sqrt{5}$. Z. Anal. Anwendungen 20 (2001), pp. 617-636.

[25] S. MaRCHI, $C^{1, \alpha}$ local regularity for the solutions of the $p$-Laplacian on the Heisenberg group for $1+\frac{1}{\sqrt{5}}<p \leq 2$. Comment. Math. Univ. Carolinae 44
(2003), pp. 33-56.

[26] A. Domokos, Differentiability of solutions for the non-degenerate p-Laplacian in the Heisenberg group. J. Differential Equations. 204 (2004), pp. 439470.

[27] A. Domokos, On the regularity of p-harmonic functions in the Heisenberg group. Ph. D. Thesis, University of Pittsburgh, 2004.

[28] J. Manfredi - G. Mingione, Regularity results for quasilinear elliptic equations in the Heisenberg group. Math. Ann. 339 (2007), pp. 485-544.

[29] G. Mingione - A. ZatorskA-Goldstein - X. ZHong, Gradient regularity for elliptic equations in the Heisenberg group. Advances in Mathematics 222 (2009), pp. 62-129.

[30] N. GarofaLO, Gradient bounds for the horizontal p-Laplacian on a Carnot group and some applications. Manuscripta Math. 130 (2009), pp. 375-385.

[31] G. Folland, Subelliptic estimates and function spaces on nilpotent Lie groups. Ark. Mat. 13 (1975), pp. 161-207.

[32] E. ACERBI - N. Fusco, Regularity for minimizers of nonquadratic functionals: the case $1<p<2$, J. Math. Anal. Appl. 140 (1989), pp. 115-135.

[33] G. Lu, Embedding theorems on Campanato-Morrey space for vector fields on Hörmander type. Approx. Theory Appl. 14 (1998), pp. 69-80.

Manoscritto pervenuto in redazione il 29 Aprile 2012. 